CÉZAR M. GERONASO DE TOLEDO

\title{
SECURE IT-SDN: A SECURE IMPLEMENTATION OF SOFTWARE DEFINED WIRELESS SENSOR NETWORK
}

Dissertação apresentada à Escola Politécnica da Universidade de São Paulo para obtenção do Título de Mestre em Ciências. 


\title{
SECURE IT-SDN: A SECURE IMPLEMENTATION OF SOFTWARE DEFINED WIRELESS SENSOR NETWORK
}

\author{
Versão Corrigida
}

Dissertação apresentada à Escola Politécnica da Universidade de São Paulo para obtenção do Título de Mestre em Ciências.

Área de Concentração:

Engenharia de Computação

Orientador:

Prof. Dr. Marcos Antonio Simplicio Junior 
Autorizo a reprodução e divulgação total ou parcial deste trabalho, por qualquer meio convencional ou eletrônico, para fins de estudo e pesquisa, desde que citada a fonte.

Este exemplar foi revisado e corrigido em relação à versão original, sob responsabilidade única do autor e com a anuência de seu orientador.

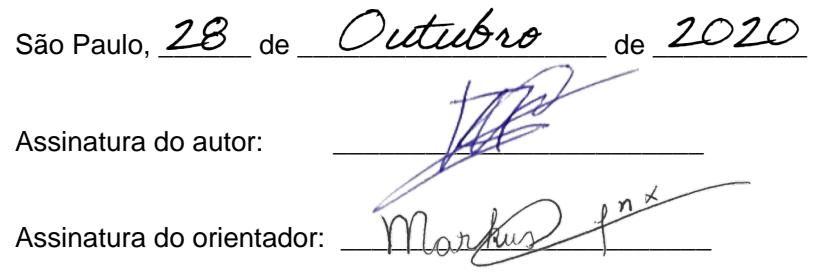

Geronaso de Toledo, Cézar Murilo

SECURE IT-SDN: A SECURE IMPLEMENTATION OF SOFTWARE

DEFINED WIRELESS SENSOR NETWORK / C. M. Geronaso de Toledo -versão corr. -- São Paulo, 2020.

$75 \mathrm{p}$.

Dissertação (Mestrado) - Escola Politécnica da Universidade de São Paulo. Departamento de Engenharia de Computação e Sistemas Digitais.

1.Wireless Software Defined Network 2.Internet of Things 3.Security I.Universidade de São Paulo. Escola Politécnica. Departamento de Engenharia de Computação e Sistemas Digitais II.t. 


\section{AGRADECIMENTOS}

Gostaria de agradecer primeiramente e principalmente a minha mãe, Inara Geronaso, por estar sempre ao meu lado em momentos difíceis e sempre me apoiando em qualquer decisão que eu tomasse. Também agradeço ao meu pai, Sérgio Rodrigues de Toledo, que apesar de não termos uma relação de muito diálogo sei que posso contar com ele para muitas coisas. E claro, não poderia deixar de agradecer a Deiser e a Mel, dois importantes membros de minha familia que não estão mais presentes para comemorar o título.

Também agradeço a todos os amigos e familiares que me apoiaram e me aturaram durante essa jornada. Também não poderia deixar de agradecer aos meus companheiros de laboratório e amigos do LARC, que me ajudaram e sempre estavam dispostos a conversar quando eu tinha dúvidas. Por fim gostaria de agradecer ao meu orientador, Marcos Antonio Simplicio Jr, que apesar de as vezes parecer distante, sempre estava disposto a conversar e me explicar as minhas repetidas dúvidas. Além disso agradeço sinceramente a oportunidade de poder realizar pesquisa no LARC.

A todas essas pessoas que passaram pela minha vida, meus sinceros agradecimentos. 


\begin{abstract}
In Software Defined Wireless Sensor Network (SDWSN), a logically centralized controller manages data flows according to high level policies, providing administrators with further control over the networks' sensor behavior. Even though the Software Defined Network (SDN) paradigm brings flexibility to how Wireless Sensor Network (WSN) are managed, it also creates security issues. After all, the controller itself and sink nodes potentially becomes a central point of failure, whereas ancillary protocols may introduce new vulnerabilities into the system. In this document, possible attacks now found at SDWSN are listed, aiming to identify the main threat vectors to which the design is exposed. As a result of this analysis, a secure framework to mitigate the attacks is presented, evaluated and prevailing challenges and future research directions are discussed.
\end{abstract}




\section{CONTENTS}

\section{List of Figures}

\section{List of Tables}

List of Abbreviations and Acronyms

1 Introduction $\quad 15$

1.1 Motivation . . . . . . . . . . . . . . 16

1.2 Goals . . . . . . . . . . . . . . . . . . 17

1.3 Method ............................. 18

1.4 Expected contributions $\ldots \ldots \ldots \ldots$

1.5 Document Organization . . . . . . . . . . . . . . . . 18

2 Background on SDN and WSN 19

2.1 SDN Architecture . . . . . . . . . . . . . . . . . . . 19

2.1 .1 SDN Controller . . . . . . . . . . . . . . . . 21

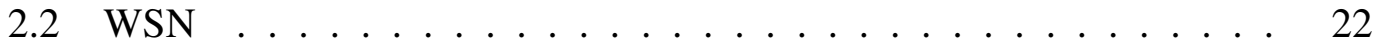

2.2.1 Routing in WSNs and Software Defined WSN (SDWSN) . . . 24

2.2.2 Security in SDWSN ................. 25

2.3 Chapter Considerations . . . . . . . . . . . . . . . . . 29 
3.1 Attacks against IT-SDN . . . . . . . . . . . 33

3.1.1 Man-in-the-Middle . . . . . . . . . . . . . . 33

3.1.2 Falsifying the Controller Node . . . . . . . . . . . . 34

3.1.3 Falsifying Sink Nodes . . . . . . . . . . . . . . 37

3.2 Chapter Considerations . . . . . . . . . . . . . . . . . 38

4 Proposed Solution $\quad 40$

4.1 Requirements . . . . . . . . . . . . . . . . . . 41

4.2 Building Blocks . . . . . . . . . . . . . . . . 42

$4.2 .1 \quad \mathrm{iSMQV} \ldots \ldots \ldots \ldots \ldots \ldots$

4.2.2 Authenticated Encryption with Associated Data . . . . . . . . 44

4.3 Secure IT-SDN . . . . . . . . . . . . . . . . . 45

4.3.1 Public/Private Key Bootstrap . . . . . . . . . . . . . . . 45

4.3.2 Symmetric Key Establishment . . . . . . . . . . . . . . . 46

4.3.3 Message format . . . . . . . . . . . . . . . . 48

4.3 .480 bits vs 128 bits $\ldots \ldots \ldots \ldots \ldots$

4.3.5 Mitigating Man-in-the-Middle attacks . . . . . . . . . 50

4.3.6 Mitigating Malicious Controller attack . . . . . . . . . . . 52

4.3.7 Mitigating Malicious Sink attack . . . . . . . . . . . . 53

$4.3 .8 \quad$ Open Issues $\ldots \ldots \ldots \ldots$

5 Experimental Evaluation $\quad 56$

5.180 bits Security Level Sinkless _ . . . . . . . . . . . . . . . 58 
5.280 bits Security Level with a Sink _ . . . . . . . . . . . . 60

5.3128 bits Security Level Sinkless _ . . . . . . . . . . . . . . . . 62

5.4128 bits Security Level with a Sink . . . . . . . . . . . . . . 63

$\begin{array}{llr}6 & \text { Final Considerations } & 67\end{array}$

6.1 Articles Published . . . . . . . . . . . . . . . . 68

References 


\section{LIST OF FIGURES}

1 Software Defined Network Model _ . . . . . . . . . . . . . . . 21

2 Wireless Sensor Network Model . . . . . . . . . . . . . . . . . . 24

3 Different routing approaches for WSNs: (I) non-SDN sensor nodes rely on SDN-ready components; (II) sensor nodes are SDN-ready; (III) WSN without SDN. Adapted from (ALVES et al., 2018). . . . . . . . 26

4 WS3N flowchart operation of the security; symmetric and asymmetric key bootstrap are depicted. Adapted from: (ALVES et al., 2018) _ . . . 28

5 Time taken by each WS3N procedure. Source: (ALVES et al., 2018) . .

6 An active Man-in-the-Middle attack against IT-SDN: the attacker can access and modify the packets' contents. . . . . . . . . . . 35

7 An attacker can map the network by eavesdropping source routed

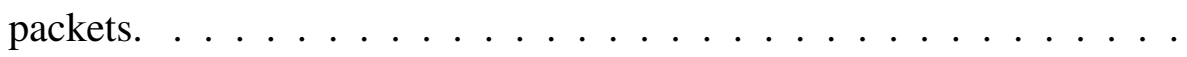

8 Falsifying the controller node: The malicious controller can corrupt all nodes on the network, except the ones past the original controller. . .

9 False sink node: a malicious sink can steal application data from nearest nodes by advertising itself as an authentic sink. . . . . . . . . 38

10 Operation modes available for the control and application planes; no security, where no security services are employed; authentication only, a MAC is used to guarantee authenticity and integrity; authenticatedencryption, AEAD scheme is employed to add confidentiality. . . . .

11 iSMQV in the IoT scenario . . . . . . . . . . . . . . . . 44 
12 The key establishment process with the controller; sensor/sink nodes start the process; after both keys are confirmed the sensor node runs its application and request flow rules to the controller. . . . . . . . . 46

13 The key establishment between sensor and sink nodes; after the sensor node requests the flow rules to the controller, the sensor node starts the AKA process with the sink; finally, after calculating the symmetric key, the sensor node can send encrypted/authenticated data. . . . . . 46

14 Packet header employed in the proposed security-enabled SDWSN ap-

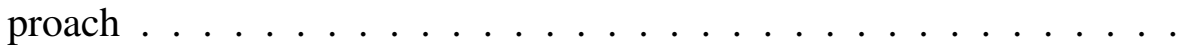

15 The internal flowchart of the AKA algorithm for 80 bits security level in the IT-SDN. The size of each packet must respect the 127 bytes limitation. . . . . . . . . . . . . . . .

16 The internal flowchart of the AKA algorithm for 128 bits security level in the IT-SDN. The 128 bits algorithm has two additional messages to respect the 127 bytes limitation. . . . . . . . . . . . . . .

17 The header after being encrypted and authenticated, the security flags are set to 11 (in binary), so the application data is encrypted and a nonce is allocated. . . . . . . . . . . . . . .

18 Man-in-the-middle mitigation: Node 3 is configured to encrypt and authenticate its applications packets before sending them; attackers are unable to eavesdrop or modify packets. . . . . . . . . . . . . . . 5

19 Process to mitigate a falsified controller: sensor node detects attacks when verifying the authenticity of public keys. . . . . . . . . . 
20 Mitigating a falsified sink node: during a data flow request, the controller checks if both source and sink nodes are authorized; if there is no authorized sink for application, the sensor node is informed to drop the packet. . . . . . . . . . . . . . . . 54

21 List of attacks mitigated and not mitigated by the proposed security

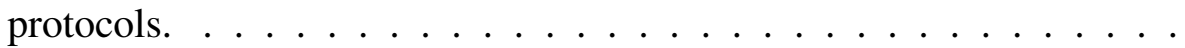

22 Time for a sensor node to execute the two most expensive procedures in the system: generating ephemeral keys and completing the whole iSMQV protocol. .................. 5

23 Percentage of nodes that completed the AKA with the controller when running the proposed security architecture with an 80-bits security level. 58

24 Delivery rate of AKA packets when establishing keys with the controller, for different network sizes. These results are from the 80 bits

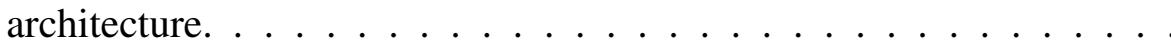

25 Time taken for the network to perform the AKA with the controller. These results are from the 80 bits architecture. Only the scenarios which completed the AKA during the simulation time are represented.

26 AKA completion rate for the network with a single sink and a single controller, for different numbers of nodes and an 80-bits security level. The curves represent the percentage nodes the controller; sink; and both completed the AKA process. . . . . . . . . . . .

27 Delivery rate of AKA packets when establishing keys with the controller and sink, for different network sizes. These results are from the 80-bits architecture. $\ldots \ldots \ldots \ldots$ 
28 Percentage of nodes that completed the AKA with the controller when running the proposed security architecture with an 128-bits security level. 63

29 Delivery rate of AKA packets when establishing keys with the controller, for different network sizes. These results are from the 128-bits architecture. ......................... 64

30 Time taken for the network to perform the AKA with the controller. These results are from the 128 bits architecture. . . . . . . . . . . 64

31 AKA completion rate for the network with a single sink and a single controller, for different numbers of nodes and an 128-bits security level. The curves represent the percentage nodes the controller; sink; and both completed the AKA process. . . . . . . . . . . 65

32 Delivery rate of AKA packets when establishing keys with the controller and sink, for different network sizes. These results are from the 128-bits architecture. Only the scenarios which completed the AKA during the simulation time are represented. . . . . . . . . . . 66 


\section{LIST OF TABLES}

1 SDN Controllers found in the literature. . . . . . . . . . . . 23

2 Solutions found in the literature and their problems . . . . . . . 26

3 Attacks against IT-SDN: a summary. . . . . . . . . . . . 38

$4 \quad$ Parameters summary for the simulations with the 80 bits framework without a Sink node. . . . . . . . . . . . . . . 57

5 Parameters summary for the simulations with an 80-bits security framework and one Sink node. . . . . . . . . . . . . . . 61

6 Parameters summary for the simulations with the 128 bits framework without a Sink node. . . . . . . . . . . . . . 63

7 Parameters summary for the simulations with the 128-bits framework with a Sink node. . . . . . . . . . . . . . . . . . 65

8 Publications during the research $\ldots \ldots \ldots 68$ 


\title{
LIST OF ABBREVIATIONS AND ACRONYMS
}

\author{
USP University of São Paulo \\ SDN Software-Defined Network \\ WSN Wireless Sensor Network \\ SDWSN Software-Defined Wireless Sensor Network \\ OF OpenFlow \\ LLDP Link Layer Discovery Protocol \\ MQV Menezes-Qu-Vanstone \\ iSMQV Implicitly Certified strengthened Menezes-Qu-Vanstone \\ IPSec IP Security Protocol \\ IoT Internet of Things \\ KGC Key Generation Center \\ SDWSN Software Defined Wireless Sensor Network \\ MitM Man-in-the-Middle \\ DoS Denial of Service \\ PKI Public Key Infrastructure
}

AKA Authenticated Key Agreement 


\section{INTRODUCTION}

In traditional networks, the switches' control guidelines are pre-installed, and play a critical role in ensuring packets are correctly and efficiently processed. As a drawback, however, managing such switches usually requires a lot of effort from network administrators. After all, every single node needs to be (possibly manually) configured in such a manner that interactions between them lead to the expected results. Often, this management overhead ends up hindering proposals aimed at improving network innovation and efficiency, which are essential to satisfy the growing diversity of consumers' needs.

Addressing this management issue is one of the promises of Software Defined Network (SDN). More precisely, SDN promises to break the aforementioned limitations of legacy networks by having a network logic decoupled from the underlying hardware and managed by a remote control plane. As a result, switches become elements without any self guidance: their only function is to efficiently redirect flows, whereas the control plane tasks are left to the network administrators. At least in principle, this precludes the need for any specific, manufacturer-dependent knowledge when configuring each switch.

Due to its prominent properties, the SDN paradigm has become more prevalent in the last years, leading to many network deployments with support to the Southbound based OpenFlow protocol (LIN et al., 2015; GUPTA; KAUR; KAUR, 2018; SHAGHAGHI et al., 2018). Unfortunately, however, the design of OpenFlow (OF) is rather oriented to- 
ward wired networks (LUO; TAN; QUEK, 2012; MAHMUD; RAHMANI, 2011; AKYILDIZ; WANG; LIN, 2016). In particular, OpenFlow assumes high-speed switches that (1) can contact the network controller as often as necessary, (2) can store (potentially large) tables with multiple flow rules, and (3) have different channels to send data and controller flows. As such, it does not necessarily cope with the needs of resource-constrained wireless environments, such as Wireless Sensor Networks (WSNs). This leads to the need of proposals able to cope not only with LLN (low power and lossy networks) protocols (e.g., IEEE 802.15.4 (MONTENEGRO et al., 2007)), but also with the limited processing power and memory availability of the network nodes. Indeed, these gaps have led to many research efforts focused on providing a Southbound protocol suitable for WSNs (ARANITI et al., 2014; ALVES et al., 2017; ALI-AHMAD et al., 2013; MAHMUD; RAHMANI, 2011).

\subsection{Motivation}

The importance of SDNs have led to many proposals in the literature aiming to identify and mitigate specific vulnerabilities and threats. Examples include denial of service (CUI et al., 2016b; CHIN et al., 2015; LIM et al., 2015), link spoofing (AZZOUNI et al., 2017; PAKZAD et al., 2014; NGUYEN; YOO, 2017), access control abuse (WEN et al., 2013; SCOTT-HAYWARD; KANE; SEZER, 2014), Man-in-the-middle (DHAWAN et al., 2015; CUI et al., 2016a). Some works also deal with specific vulnerabilities on an SDN environment, such as vulnerable libraries on controllers (MEDVED et al., 2014; PICKETT, 2014), or the detection of SYN flood-in attacks at the control plane (MOHAMMADI; JAVIDAN; CONTI, 2017) Furthermore, there are many studies focused on security aspects of WSNs, taking into account their inherent resource limitation. Some examples are proposals and surveys that discuss sink holes (KAVITHA; SRIDHARAN, 2010; SHARMA; GHOSE, 2010), denial of service (PELECHRINIS; ILIOFOTOU; KRISHNAMURTHY, 2011; MO et al., 2010), man-in-the-middle (KAVITHA; SRIDHARAN, 2010; 
AL AMEEN; LIU; KWAK, 2012), and different aspects of cryptography (SIMPLICIO JR et al., 2017; SIMPLICIO et al., 2013a; SIMPLICIO et al., 2010).

Despite the importance of such works on SDNs and WSNs as separate topics, studies that consider their combination have become more prevalent only in the past few years. Commonly, their goal is to employ the SDN paradigm in WSNs, creating a Software-Defined Wireless Sensor Network (SDWSN), thus providing dynamic and scalable routing algorithms for accommodating the different needs of WSN applications. One example of such combination is the IT-SDN (ALVES et al., 2017; MARGI et al., 2018), which is the only solution found in the literature that discuss performance analysis of larger network sizes. IT-SDN, also, is the only solution that uses source routed control packets to relieve network overhead, taking into account the limited resources of a WSN node. Albeit promising, SDWSNs such as IT-SDN are prone to security vulnerabilities inherited from SDNs and also from WSNs, leading to the need of a robust security framework for addressing them.

\subsection{Goals}

The main goal of this work is to design a secure framework for SDWSNs. In particular, we focus on the open-source IT-SDN (ALVES et al., 2017; MARGI et al., 2018) solution, addressing classical security issues and also specific vulnerabilities found in the SDWSN scenario. The proposed design leverages lightweight cryptographic solutions, such as iSMQV (SIMPLICIO JR et al., 2017), as well as standardized and widely adopted protocolos, such as IPSec (FRANKEL; KRISHNAN, 2011). It is important to note that, this solution does not develop new protocols or algorithms. It uses the building blocks above to mitigate security problems. 


\subsection{Method}

The development of this work starts with a comprehensive research on security issues in the context of SDNs, focusing on how these vulnerabilities apply and/or are amplified in a WSN environment. By surveying and organizing the identified security issues, a threat classification can be drawn. The resulting classification can then be employed to evaluate how the coverage of existing proposals, thus identifying gaps that could lead to the proposal of new solutions. The solution hereby proposed, building upon cryptographic guidelines that take into account WSN limitations, aims specifically to address the identified gaps. Finally, the solution is implemented, tested, and evaluated in terms of security and performance.

\subsection{Expected contributions}

The main contribution of this work is the proposal of a security framework for SDWSNs, addressing essential security issues identified in the literature, as well as its deployment and evaluation on the Cooja (OSTERLIND et al., 2006) simulator.

\subsection{Document Organization}

This document is organized in chapters. Chapter 2 covers this work's theoretical background, focusing on important aspects of SDNs and WSNs. It also analyses the main security issues in SDWSNs, proposing a threat classification that allows the identification of gaps. Chapter 3 describes the IT-SDN framework for enabling SDWSNs. Chapter 4 presents the proposed security architecture for addressing the threats identified, building upon on IT-SDN for creating a concrete, secure SDWSN instance. Chapter 5 shows the results of the simulations with the proposed solution. Finally, Chapter 6 presents our final considerations and next steps, also listing envisioned and published publications resulting from this work. 


\section{BACKGROUND ON SDN AND WSN}

This chapter presents a conceptual and technical background on software-defined networking. It also discusses the concept of wireless sensor networks (WSN), and their importance in the context of Internet of Things (IoT).

\subsection{SDN Architecture}

The term SDN appeared in (GREENE, 2009), referring to the ability of OpenFlow (MCKEOWN et al., 2008) to support the configuration of table flows in routers and switches using software. However, the ideas behind SDNs come from the goal of having a programmable network. Today, the networking industry has shown enormous interest in the SDN paradigm. This interest comes mainly from the expectations of reducing capital and operational costs with service providers and enterprise data center, which can be achieved with programmable, virtualizable and easily partitionable networks. In reality, programmability is also becoming a strategic feature for network hardware vendors, since it allows many devices to be programmed and orchestrated in large network deployments.

As briefly mentioned in Chapter 1, the SDN paradigm gives administrators further control over the network elements' behavior. To accomplish this, SDN presents three planes that are logically connected: the data, control, and application planes. The data plane is where the switches are located, and its only function is forwarding packets. The control plane is where the controller that manages all network logic is located, 
defining how packets should be handled by each switch. And the application plane is where users interact with the controller, developing applications and managing the state of the network. An example of interaction between these layers is an application that can read the packet statistics of a switch to detect an attack in the data plane, and then change the rules located in the control plane to mitigate this attack.

In SDN, external modules interacting with the controller can be logically divided in 4 parts, depending on the "direction" from which the interaction occurs (see Figure 1):

- Southbound Interface: handles the connection between the controller and network's forwarding elements in the data plane (e.g., switches). The current trend in the southbound interface is to use the OpenFlow protocol. This protocol is, thus, responsible for tasks like installing flows on the switches, following the controller's policies, as well as reporting flow statistics and architecture changes to the controller, so the latter has a global view of the network's state.

- Northbound Interface: All types of applications that want to interact with the controller (and, indirectly, with the underlying network and traffic) are typically designed in this plane. Unlike the southbound interface, there is currently no widely standardized protocol for the northbound interface. Some efforts do exist in that sense, though, such as using a REST API. However, some challenges still exist for the adoption of this (or, actually, any other) approach for standardization. Examples include the security of the communication with the controller, the handshaking methods between the northbound applications and the controller, how to manage communicative permissions or authorization, and how to handle conflicts among different applications (PORRAS et al., 2012; SON et al., 2013).

- East and West Interface: These interfaces are absent in some SDN architectures due to the fact that they are solely used for managing distributed SDNs. For 


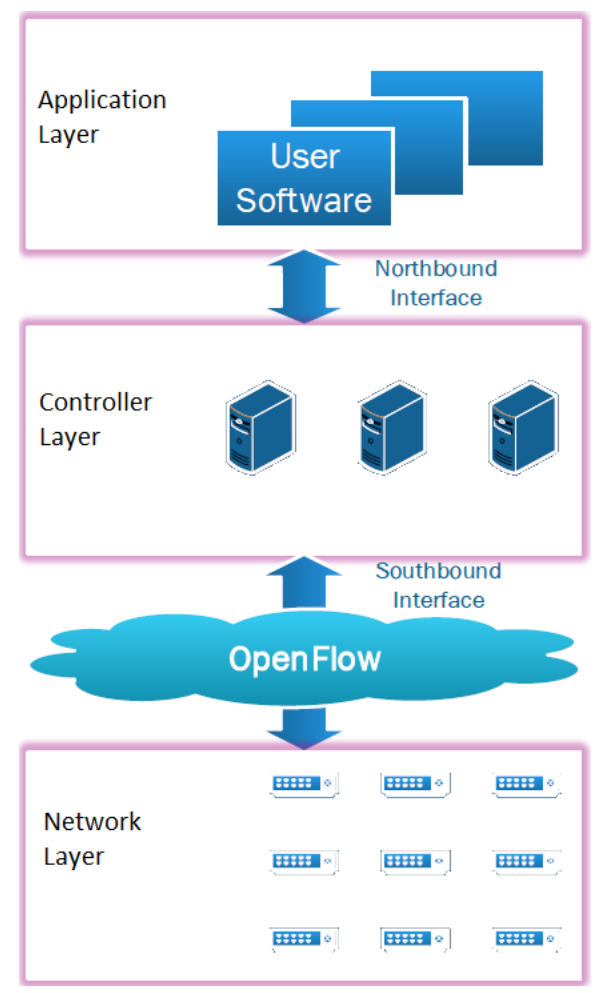

Figure 1: Software Defined Network Model

example, different instances of controllers in a distributed architecture should communicate frequently for exchanging control and management information. This could be achieved, for example, by means of a hierarchical implementation where a "main controller" manages several lower-level controllers, defining their access privileges in the network (SCHMID; SUOMELA, 2013).

\subsubsection{SDN Controller}

The SDN controller, which is also called a network operating system, is the module upon which all network control applications are deployed. Hence, it is the entity responsible for defining the switches' behavior. When the OpenFlow protocol is adopted, for example, this is done by installing flow rules in each switch. More precisely, when a switch receives a packet for which no rule is installed, the switch encapsulates that packet as a "packet-in" message and sends it to a controller. The controller, based on its current configurations, decides which should be the treatment given to that flow; exam- 
ples include dropping the packet, forwarding it, modifying its header, or keep sending that type of packets to the controller. This decision is sent to all relevant switches in the network via a "packet-out" message; e.g., if the packet should be forwarded to its destination, then the flow tables on all switches along the packet's path are configured accordingly.

Typical SDN controller implementations contain a basic set of services, such as routing, multicasting, security, access control, bandwidth management, node discovery, traffic engineering and all forms of policy management. (SHERWOOD et al., 2009; MEDVED et al., 2014; DHAWAN et al., 2015; FLOODLIGHT, 2017) The controller is not limited to redirecting flows, though, since new services can be enabled using specialpurpose applications installed on it (e.g., applications developed using the P4 SDNoriented programming language (BOSSHART et al., 2014)).

Given this central role of the controller in SDNs, it must be developed to be flexible, available, secure and scalable. After all, if any data plane component stops working correctly, the controller can reprogram the network to avoid the faulty elements. However, if the controller itself fails, the network becomes unable to handle packets for which no instruction has been given (by the controller) in the past. For this reason, many groups have focused exactly in the development of robust SDN controllers (PAULISTA, 2017). Table I shows the characteristics of some of the main controllers currently available.

\subsection{WSN}

A WSN can be defined as a network of tiny micro-electronic devices, called sensor nodes or motes, that gather information from the environment and exchange it among themselves and with other network elements via wireless links. Usually, the nodes are spatially distributed, so the information of a larger area can be cooperatively acquired. 
Table 1: SDN Controllers found in the literature.

\begin{tabular}{|c|c|c|}
\hline Controller & Language & Description \\
\hline Nox & $\mathrm{C}$ & $\begin{array}{l}\text { First OpenFlow Controller, it works as Openflow } \\
\text { controller or OpenFlow switch }\end{array}$ \\
\hline Pox & Python & SDN controller via OpenFlow protocol \\
\hline Maestro & Java & $\begin{array}{l}\text { SDN controller supports multi-threading } \\
\text { and parallelization management }\end{array}$ \\
\hline Ryu & Python & SDN controller supports OpenFlow v1.0, and v1.2 to v1.5 \\
\hline MUL & $\mathrm{C}++$ & SDN controller supports Openflow v1.0, v1.3, v1.4 and Ovsdb \\
\hline Beacon & Java & OpenFlow controller supports threaded operation \\
\hline Floodlight & Java & $\begin{array}{l}\text { OpenFlow controller supports multi-threading } \\
\text { and hybrid networks (SDN + traditional switches) }\end{array}$ \\
\hline IRIS & Java & $\begin{array}{l}\text { OpenFlow controller supports multi-domain } \\
\text { and Openflow protocols v1.0-v1.3 }\end{array}$ \\
\hline OESS & Perl & $\begin{array}{l}\text { OpenFlow controller supports sub-second } \\
\text { circuit provisioning and automatic failover }\end{array}$ \\
\hline NodeFlow & Javascript & OpenFlow controller for Node.js \\
\hline Ovs-controller & $\mathrm{C}++$ & Simple OpenFlow controller \\
\hline Flowvisor & $\mathrm{C}++$ & $\begin{array}{l}\text { OpenFlow controller acts as a transparent } \\
\text { proxy between switches and multiple controllers }\end{array}$ \\
\hline RouteFlow & $\mathrm{C}++$ & $\begin{array}{l}\text { OpenFlow controller provides } \\
\text { virtual Internet Protocol routing servers }\end{array}$ \\
\hline OpenDaylight & Java & $\begin{array}{l}\text { SDN controller supports scalability, multi-protocol } \\
\text { infrastructure and heterogeneous multi-vendors networks }\end{array}$ \\
\hline
\end{tabular}

The collected data is then sent to a special node, called sink, which can use the data locally (e.g., issuing commands to an actuator) and/or send it to other networks. For instance, in an autonomous irrigation system, the sink may run an application that, based on the current humidity data collected by sensors, sends commands to a sprinkler connected to it. The sink can connect to the Internet and send the collected data to a 


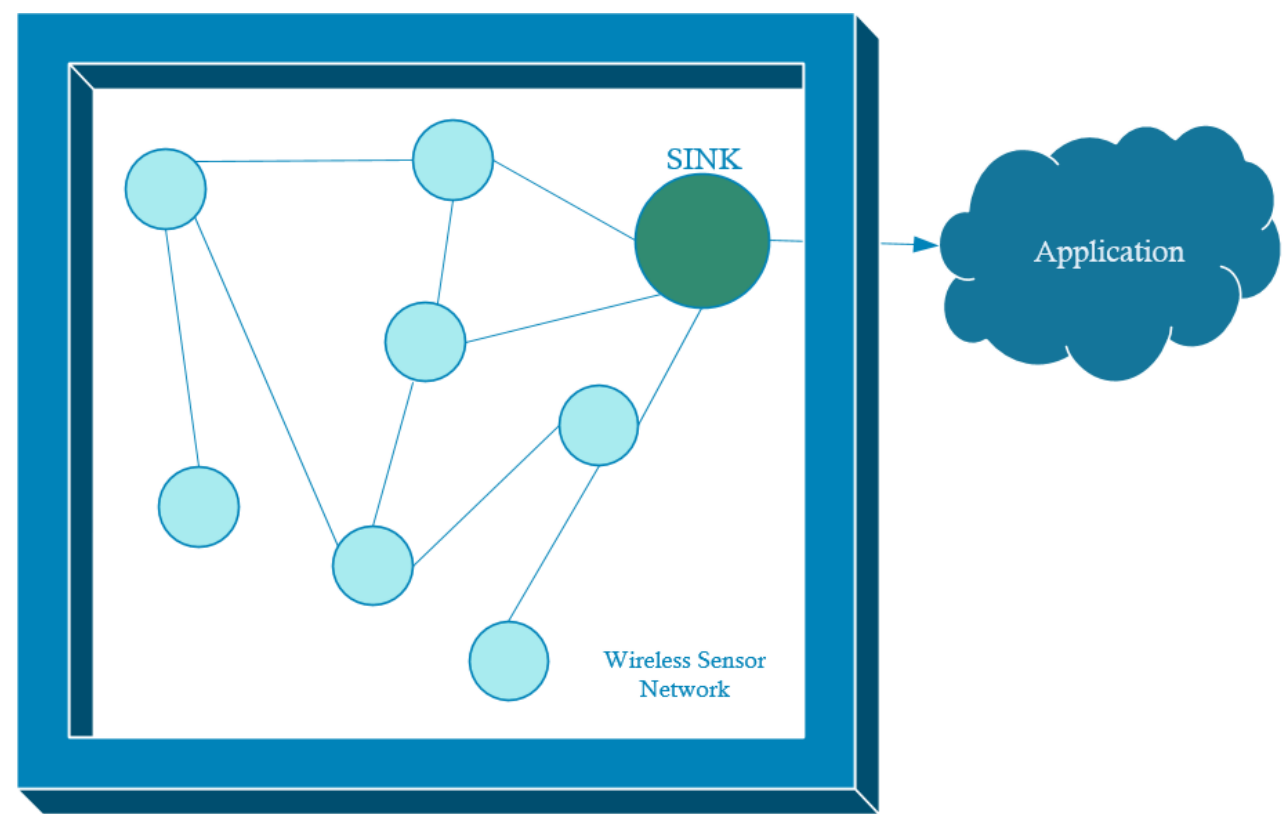

Figure 2: Wireless Sensor Network Model

remote database, so it may be used for research and forecasting. In the Internet of Things (IoT) context, WSN play, thus, an important role: they help users (humans or machines) to interact with their environment and react to real-world events.

Figure 2 shows the overall topology of a typical WSN, in which sensor nodes form a mesh network and send the collected data to a sink, which can then share this data with external applications.

\subsubsection{Routing in WSNs and Software Defined WSN (SDWSN)}

The manner by which packets are routed in WSNs is not bound by any standard. More precisely, even though some standards do exist (e.g., Routing Protocol for Low power and Lossy Networks - RPL (WINTER et al., 2012)), each WSN deployment is likely to adopt routing algorithms aimed at optimizing different metrics, such as energy usage, latency or reliability. Therefore, the task of choosing a suitable routing protocol is highly application-dependent. Unfortunately, though, this creates a complex environment for developers and network administrators, in particular when multiple applications running on the same WSN have conflicting requirements in terms of 
which metric(s) should be optimized.

One of the goals of the Software Defined WSN (SDWSN) paradigm is to address this complexity, conciliating the different routing approaches via the network controller. Following this promise, SDWSN has been a trending research topic in the past few years (FLAUZAC et al., 2015; TADINADA, 2014; MAHMUD; RAHMANI, 2011; DE OLIVEIRA; GABRIEL; MARGI, 2015). One of the current efforts in the area is to specify a suitable southbound interface. This is a challenging issue, though, in particular because the main protocol employed for this purpose on wired networks (OpenFlow) is not very well adapted to highly dynamic and user-dense wireless networks (LUO; TAN; QUEK, 2012). In addition, at least in principle OpenFlow was not designed for highly constrained network nodes, where resources such as energy, processing, memory and communication are very limited. Since WSNs commonly combine these characteristics, new approaches are likely to be needed for the deployment of SDWSN.

Analyzing the literature, we were able to identify two main approaches for SDWSN. In a first approach, the sensor nodes are seen as non-SDN components, so they must rely on SDN-capable devices deployed in the same network (FLAUZAC et al., 2015; TADINADA, 2014). Conversely, a second approach considers WSN nodes as SDNready, so no "extra" components are required (MAHMUD; RAHMANI, 2011; LUO; TAN; QUEK, 2012; GALLUCCIO et al., 2015; DE OLIVEIRA; GABRIEL; MARGI, 2015; ARANITI et al., 2014; ALVES et al., 2017; ALVES et al., 2018). Figure 3 depicts these approaches, comparing them with a regular, non-SDN based WSN.

\subsubsection{Security in SDWSN}

As could be expected from an emerging model, some aspects of SDWSNs are still not formally specified. One of those aspects, which is of particular interest in this work, is security. To consider security on SDWSN we have to take into account two important factors; (1) the resource constraint aspect of WSN, taking into consideration the 

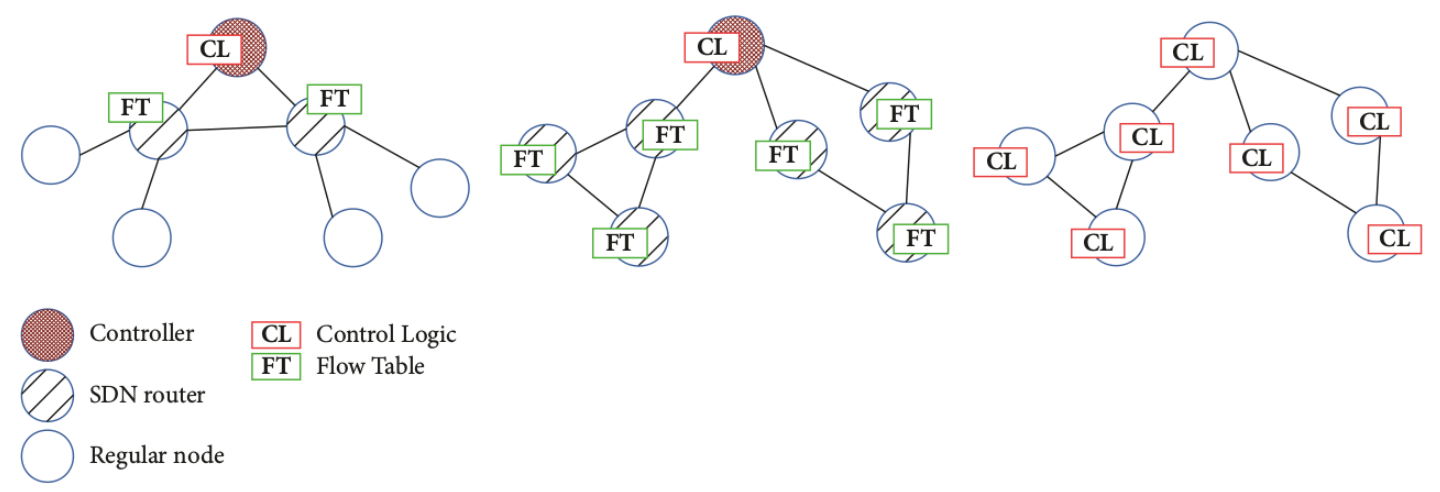

Figure 3: Different routing approaches for WSNs: (I) non-SDN sensor nodes rely on SDN-ready components; (II) sensor nodes are SDN-ready; (III) WSN without SDN. Adapted from (ALVES et al., 2018).

Table 2: Solutions found in the literature and their problems

\begin{tabular}{ll}
\hline Solution & Problem \\
\hline Sensor Openflow & Uses Openflow \\
Exploitation of Openflow in WSN & Uses Openflow \\
SDN-Wise & Show results of only 2 sensor nodes, \\
Framework for IoT based on 6lowpan & does not consider larger networks \\
& Does not has an implementation
\end{tabular}

802.15.4 specification and (2) the wireless aspect of WSN. Some solutions are based on TLS provided by the OpenFlow protocol (LUO; TAN; QUEK, 2012; MAHMUD; RAHMANI, 2011; ALQALLAF; WANG, 2015; PRITCHARD; HANCKE; ABU-MAHFOUZ, 2017), as discussed before this is not ideal. Others solution only mention and does not detail the security aspect (JARARWEH et al., 2015; SAHOO; SAHOO; PANDA, 2015; MARGI et al., 2018). Table 2 shows why the solutions found in the literature are not ideal.

Indeed, to the best of our knowledge, WS3N (ALVES et al., 2018) is one of the few works in the literature that deals with security issues in SDWSN. Basically, WS3N's security infrastructure comprises an Authorization server and a Key Generation Center (KGC). The Authorization server is responsible for managing the database of authorized sensor nodes, informing the KGC of their authorization status. The KGC, in turn, is responsible for issuing digital certificates to authorized nodes. Nodes bootstrapped by the KGC can then establish a secure communication channel with the controller 
using an authenticated key agreement (AKA) protocol. This process is illustrated in Figure 4, and can be summarized by the following steps:

1. The controller and sensor nodes run the neighbor discovery protocol at the startup of the network.

2. Controller installs flow rules in the sensor nodes, so they know how to reach the authentication server.

3. The sensor node that wants to join the network sends its admission ID to the Authentication Server.

4. Authentication Server checks in its database to see if the sensor node is authorized; if that is the case, the controller is informed that it can proceed.

5. The controller installs flow rules in the authorized node, so it learns how to reach the KGC.

6. The SDN-ready sensor node sends a public-key bootstrap request to the KGC, using iSMQV (SIMPLICIO JR et al., 2017).

7. KGC also checks the node's authorization status with the Authentication Server.

8. KGC, after checking the aforementioned authorization status, sends the AKA bootstrap response containing the information required for the node to compute a valid public-private key pair.

9. After obtaining the asymmetric key, the node can perform symmetric key exchanges with all nodes in the network.

The AKA scheme adopted by WS3N, iSMQV (SIMPLICIO JR et al., 2017) was designed specifically with WSN in mind. Basically, it comprises two mechanisms. The first consists in a security bootstrap protocol that enables nodes to get implicitly certified public-private key pairs (BROWN; GALLANT; VANSTONE, 2002; CAMPAGNA, 2008), 


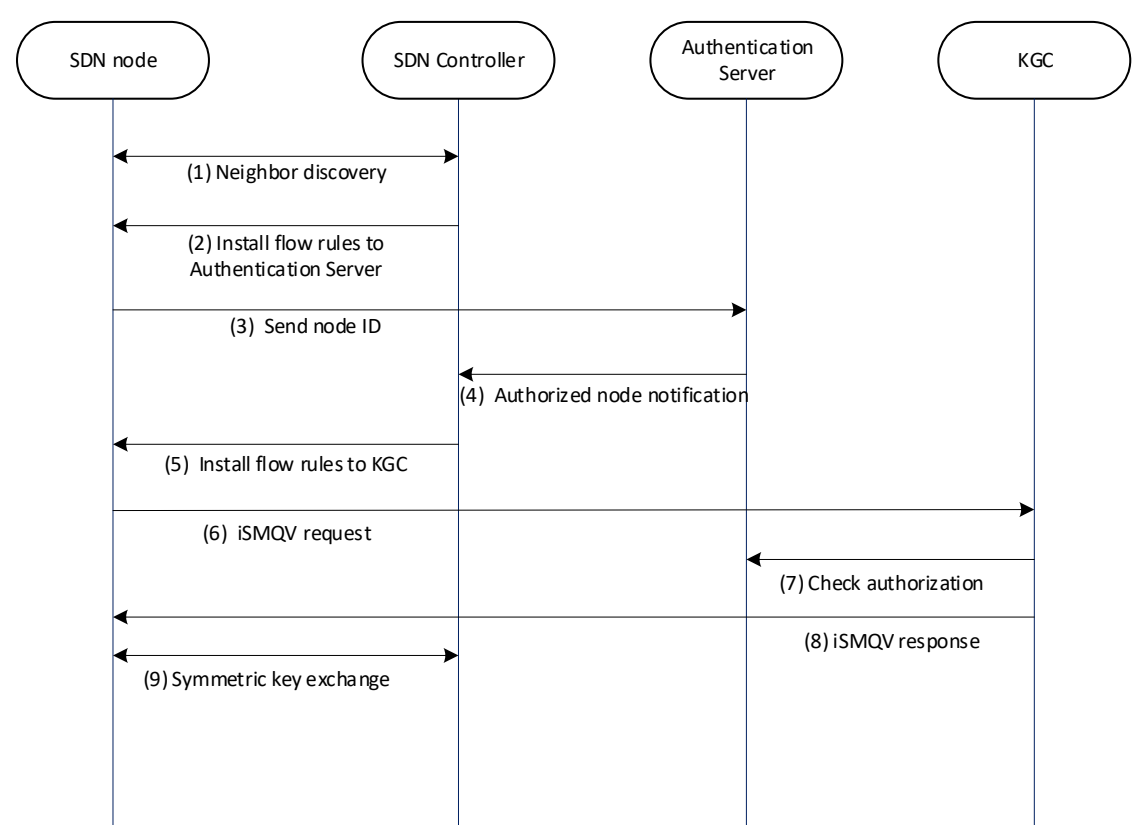

Figure 4: WS3N flowchart operation of the security; symmetric and asymmetric key bootstrap are depicted. Adapted from: (ALVES et al., 2018)

avoiding the transmission of large certificates commonly used in traditional networks. These keys are then used in a lightweight authenticated key agreement protocol, so any node can establish secret and authenticated key pairs with other nodes. In addition, iSMQV is escrow-free, i.e., the trusted authority does not learn the private keys of all nodes.

Even though iSMQV enables a reasonably efficient key agreement process in WS3N, the architecture's overall scalability could be improved. More precisely, as the number of nodes participating in the WS3N network increases, the additional control traffic and consequent collisions and buffer overflows lead to a high packet drop rate. For example, according to the experiments in (ALVES et al., 2018), the delivery rate for data packets is lower than $10 \%$ for a scenario with more than 64 nodes that uses the ContikiMAC MAC protocol. Also, if every new flow creates an extra flowtable entry in each node, that table would overflow; the node would then restart and become inaccessible for some time. 
The bootstrap procedure in WS3N is also responsible for considerable delays to the network's operation. Indeed, as shown in Figure 5, the time for each procedure (authorization, asymmetric key bootstrap and symmetric key agreement) ranges from 55 and 350 seconds depending on the number of nodes. Therefore, new nodes trying to send application data fail to do so until they receive directions from the controller. This is particularly troublesome considering that the reference implementation uses 80-bits security level (RESEARCH, 2000), less than the mininum recommended security level specified by the United States National Institute of Standards and Technology (NIST) (National Institute of Standards and Technology, 2013), which is currently at 112 bits. Hence, a higher security level should lead to an even lower efficiency, however, it should be necessary if security must be achieved. All in all, and even though WS3N has the merit of bringing security rules to the SDWSN environment, the current architecture can still be improved.

\subsection{Chapter Considerations}

SDN is an interesting paradigm to use with WSN, in particular because SDN centralizes packet routing directives in a more robust node: the controller. As a result, sensor nodes do not need to handle route management and, thus, can concentrate their (limited) resources on application-related tasks.

Despite this promise of SDWSN, enforcing security in this environment is still an open challenge. Indeed, one of the few solutions available in the literature, WS3N, leads to a noticeable degradation of network' quality, in special when the number of nodes is high. Nevertheless, this does not mean that WS3N's internal mechanisms are themselves a problem, but simply that there may be a way to integrate similar integrity, authenticity and confidentiality mechanisms into a SDWSN architecture. With this goal in mind, the next chapter analyses the security of a more efficient SDWSN solution, IT-SDN (ALVES et al., 2017), which is then used as basis for the security-enabled 


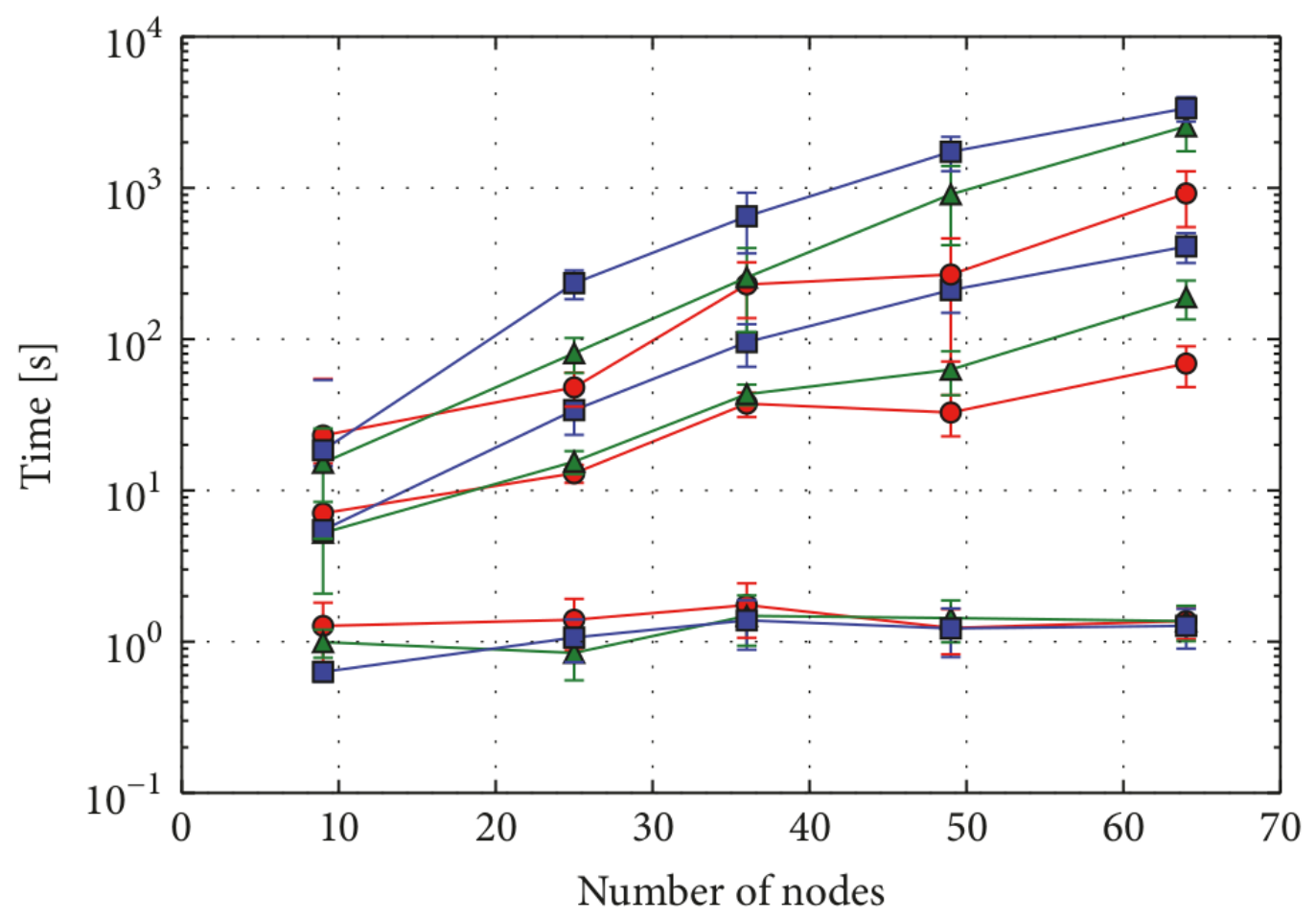

- - Auth ContikiMAC

$\Delta \Delta$ Bootstrap ContikiMAC

a- Key agreement ContikiMAC

Figure 5: Time taken by each WS3N procedure. Source: (ALVES et al., 2018)

SDWSN proposed in the subsequent Chapter 4. 


\section{IT-SDN}

IT-SDN (ALVES et al., 2017) is an open tool inspired by TinySDN (DE OLIVEIRA; GABRIEL; MARGI, 2015). IT-SDN has three main communication protocols: southbound, neighbor discovery and controller discovery. By employing source-routed control packets, it can reduce overheads and flow table occupancy, which facilitates largescale deployment. The packets in the IT-SDN framework are limited to 127 bytes, this choice was made to respect the 802.15.4 standard. Although IT-SDN has its own feature, it uses some from the contiki operating system (DUNKELS; GRONVALL; VOIGT, 2004), namely the radio communication protocol.

According to the IT-SDN design, all sensor nodes are SDN-enabled, and there is at least one controller among the nodes. The routing of the packets is done according to its corresponding flow identification number (flow id), defined by the application that generated it. Therefore, each type of packet has one specific destination node according to its flow id, so it is the controller's responsibility to route the flow to the sink. By using this concept, the network may accommodate multiple sinks, and there is no need for the controller and the sink to be executed on the same device.

IT-SDN has its own Southbound protocol specification, composed of seven packet types, defined as follows:

- Flow request: packets a node send to the controller for requesting flow entries, so it learns how to route the packet. 
- Flow setup: packets sent by the controller to configure the flow tables on the sensors. These packets may be transmitted in response to flow request packets or due to route recalculations performed by the controller. One particularity of IT-SDN is that the controller can use three flow setup types, either using a preconfigured rule or an application installed in the controller's core: regular, source routed and multiple.

- Regular: the regular flow setup is found in common SDN setups. It configures the flow table of all intermediate nodes, from the packet's origin to its destination.

- Source routed: contains the route to the requesting node in the packet header. The flow setup packet is then source routed, so there is no need to configure intermediate nodes' flow tables.

- Multiple flow: is used to update as many nodes as possible with a single packet. In this case, a single packet traverses the network deploying flow rules in each sensor node it goes through.

- Flow ID register: packets used to inform the controller of a destination candidate for the specified flow id. It can be used, for example, by a sink node when telling the controller that it is the target of some application's flow ID.

- Acknowledgement: packet sent by sensor nodes for confirming the delivery of control packets.

- Data packet: application layer packets that are resolved by applications at the northbound interface.

- Neighbor discovery / controller discovery: used by the underlying Neighbor discovery and Controller discovery protocol. Currently there is no formalization on how these packets should be implemented (CHEN; BIAN, 2016), so IT-SDN 
is not bound to any specific protocol. Instead, interfaces are defined to enable implementing and choosing between alternatives. The ND protocol is responsible for calculating the link metrics, such as hop count, Received Signal Strength Identification (RSSI), and Expected Transmission Count (ETX).

- Neighbor report: contains node neighborhood information. It is employed by the neighbor discovery protocol request, so every time there is a change in a node's neighborhood, it notifies the controller.

By enforcing source-routed control packets, IT-SDN can achieve a delivery rate of almost $100 \%$ of the control packets for a network composed of 64 nodes (MARGI et al., 2018). Assuming we can leverage such scalability property, this makes IT-SDN a compelling candidate for use as basis for the proposed security architecture. As a first step toward this goal, thus, we must consider which type of attacks can be performed against the IT-SDN framework.

\subsection{Attacks against IT-SDN}

The main vulnerability found in IT-SDN is that it includes no authentication and authorization mechanisms, meaning that new nodes (including attacker nodes) can be added at will. In this section, we build upon this issue and show three types of attacks that can be performed against the IT-SDN architecture, as well as their potential consequences.

\subsubsection{Man-in-the-Middle}

We start with a classical, Man-in-the-middle (MitM) attack, i.e., an attack in which an intruder controls all traffic between two honest network nodes. As a result, the intruder can inject falsified data in the communication, as well as eavesdrop all information exchanged between the victims. Therefore, attackers can violate not only the 
communications' integrity and authenticity, but also break its confidentiality for learning application data (e.g., readings sent to a sink) and control information (e.g., the network's topology and policies). In particular, the source-routed nature of IT-SDN is expected to give interesting information about existing links among network nodes, as well as about which are preferential routes for each network source and/or destination. As a result, the attacker can build an accurate network fingerprint (CUI et al., 2016a). Such a fingerprint might be useful, for example, for building other types of attacks, such as isolating some network nodes by taking down their neighbors, thus affecting the network's availability. Those node's data can then be replaced with the attacker's forged data, thus affecting the system's behavior.

Figure 6 shows a typical active MitM attack in a SDWSN scenario. In this figure, node 3 generates a flow that is intended to go to the sink $\alpha$, but an attacker is among the nodes in the route defined by the controller. As a result, the attacker can capture the packets from this flow, drop some of them, and/or insert its own packets in the communication.

In addition, Figure 7 shows a passive MitM attack, in which the attacker's goal is simply to fingerprint the network. Specifically, by verifying the routes listed on the header of any source-routed flow, the attacker identifies which nodes are directly connected; e.g., in the examples shown in the figure, it learns that node \#9 knows how to reach node \#5 and that the latter knows how to reach node \#3.

\subsubsection{Falsifying the Controller Node}

It is possible to falsify IT-SDN's controller node, although such an attack is not as simple as falsifying a sensor node. Specifically, to perform this attack, the intruder first needs to have the IT-SDN specification of the controller. In possession of this information, the attacker can interfere with the very first process that occurs when a node joins the network: it looks for a viable route for the controller. Specifically, if the 


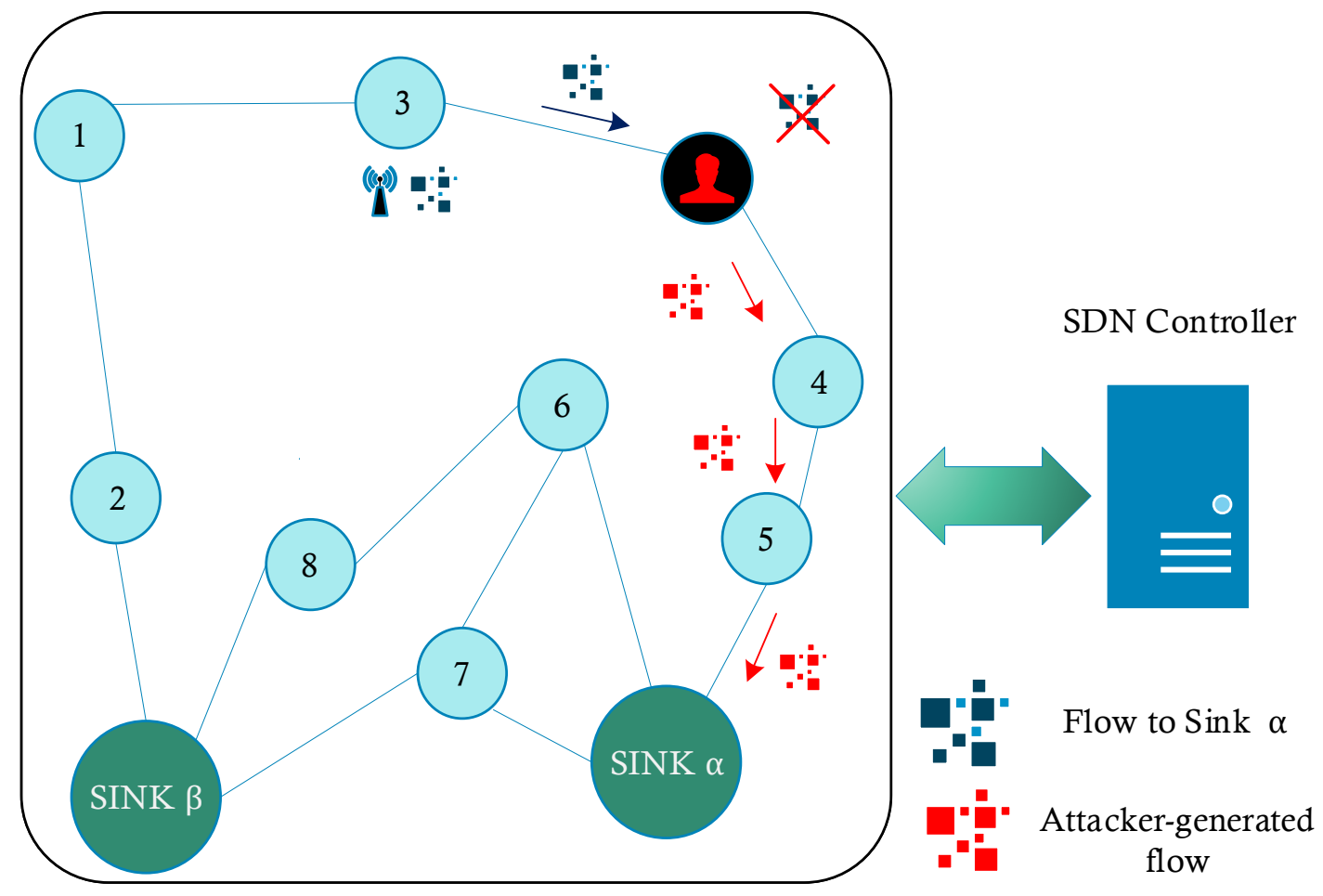

Figure 6: An active Man-in-the-Middle attack against IT-SDN: the attacker can access and modify the packets' contents.

malicious node announces itself as the network controller, the victim is unable to notice that this information is actually fake, and accept any controller rules from the attacker. After all, IT-SDN's controller discovery protocol is implemented in such a manner the nodes broadcasts its informations through a neighbour report packet to nearby nodes until it reaches the controller. The controller after receiving this packet, will set the route in each sensor node of how to reach itself, nearby nodes acknowledge it as an SDN controller. However a malicious controller can configure for its neighbour nodes to relay the packet that configures the route to the controller. This process is repeated until all nodes have a route to the malicious controller.

Figure 8 shows an example of a malicious controller attack. As illustrated in this figure, the malicious controller can run the neighbor discovery protocol when it enters the network. Since sensor nodes are configured to accept any new controller on the 


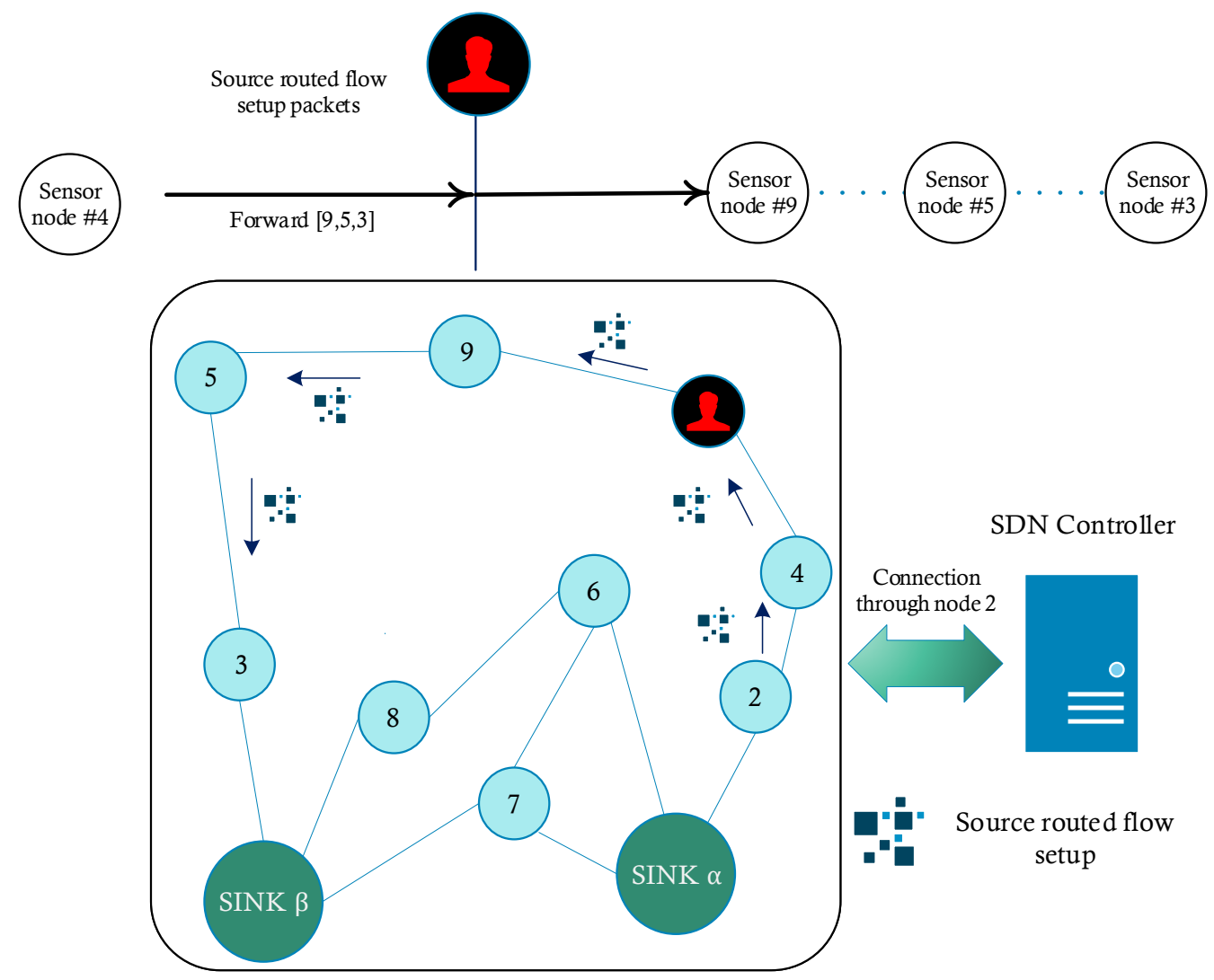

Figure 7: An attacker can map the network by eavesdropping source routed packets.

network, the attacker is able to take over all of their (direct or indirect) neighbors. The only exception are the nodes connected to the original controller. The reason is that the controller node is not configured to receive other controllers' notifications. Hence, the controller discovery packet stops at the controller node, so nodes that only have communication through the original controller should not be corrupted.

Besides creating a DoS effect from the original controller's perspective, the malicious controller can install any flow rules in the network's sensor nodes. As a result, the attacker can, for example, steal any application data and/or stop specifics nodes from receiving data. 


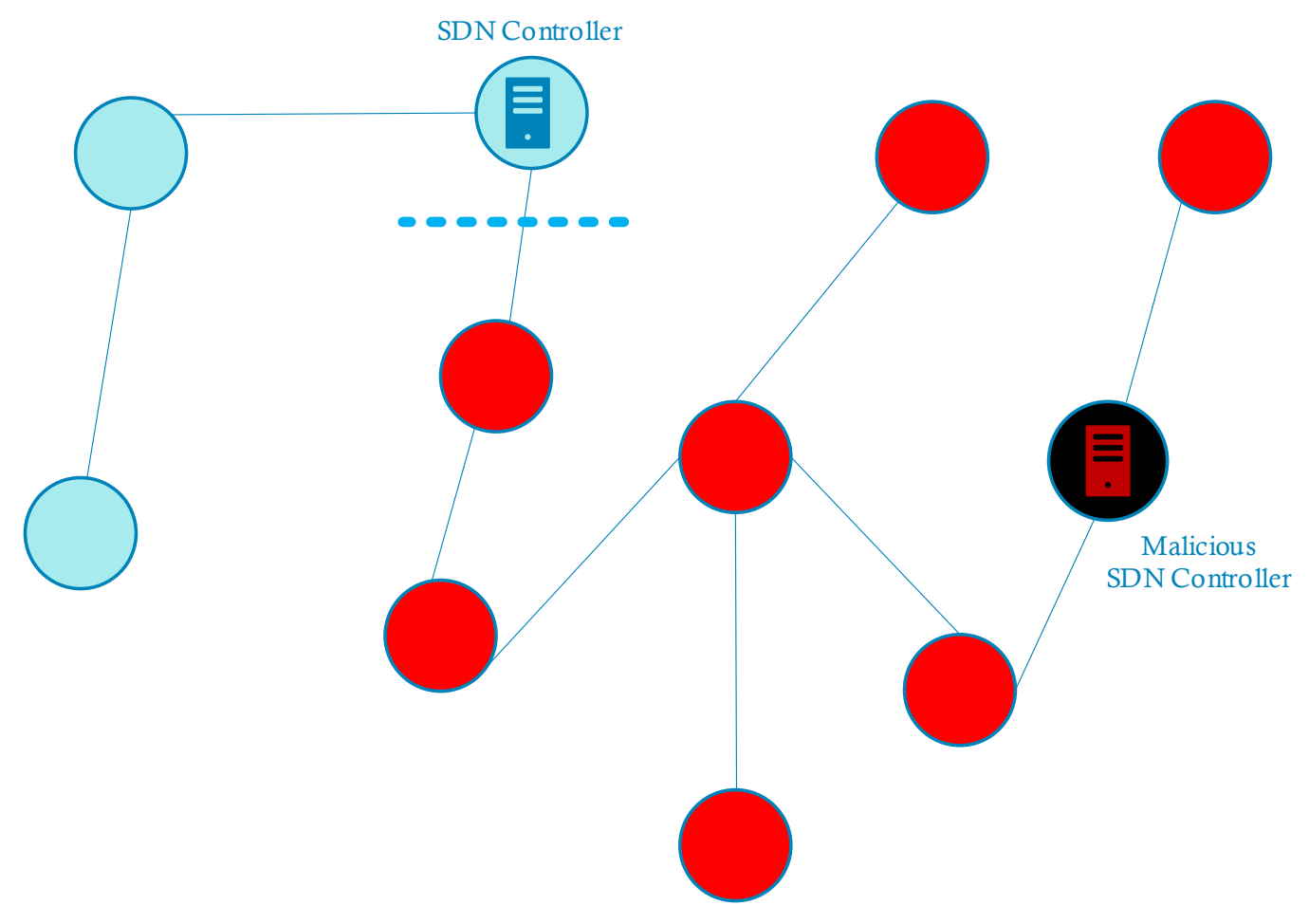

Figure 8: Falsifying the controller node: The malicious controller can corrupt all nodes on the network, except the ones past the original controller.

\subsubsection{Falsifying Sink Nodes}

IT-SDN routing guidelines rely on flow identifiers (flow-id). It also supports multiple sinks for the same flow-id, and the controller is expected to adjust the network's routing behavior so that all packets are delivered to the nearest sink. In this scenario, the lack of authentication mechanisms allows a false sink node to advertise itself as one of the targets for a specific flow-id. Consequently, such a malicious sink gains access to the application's intended data, creating a sinkhole (NGAI; LIU; LYU, 2006) in the network.

The example in Figure 9 shows the fragmentation of the network caused by sinkhole attack. Namely, blue nodes are assumed to be near the original sink, so they remain unaffected by the attack, whereas red nodes have their traffic directed to the 
malicious sink close to them.

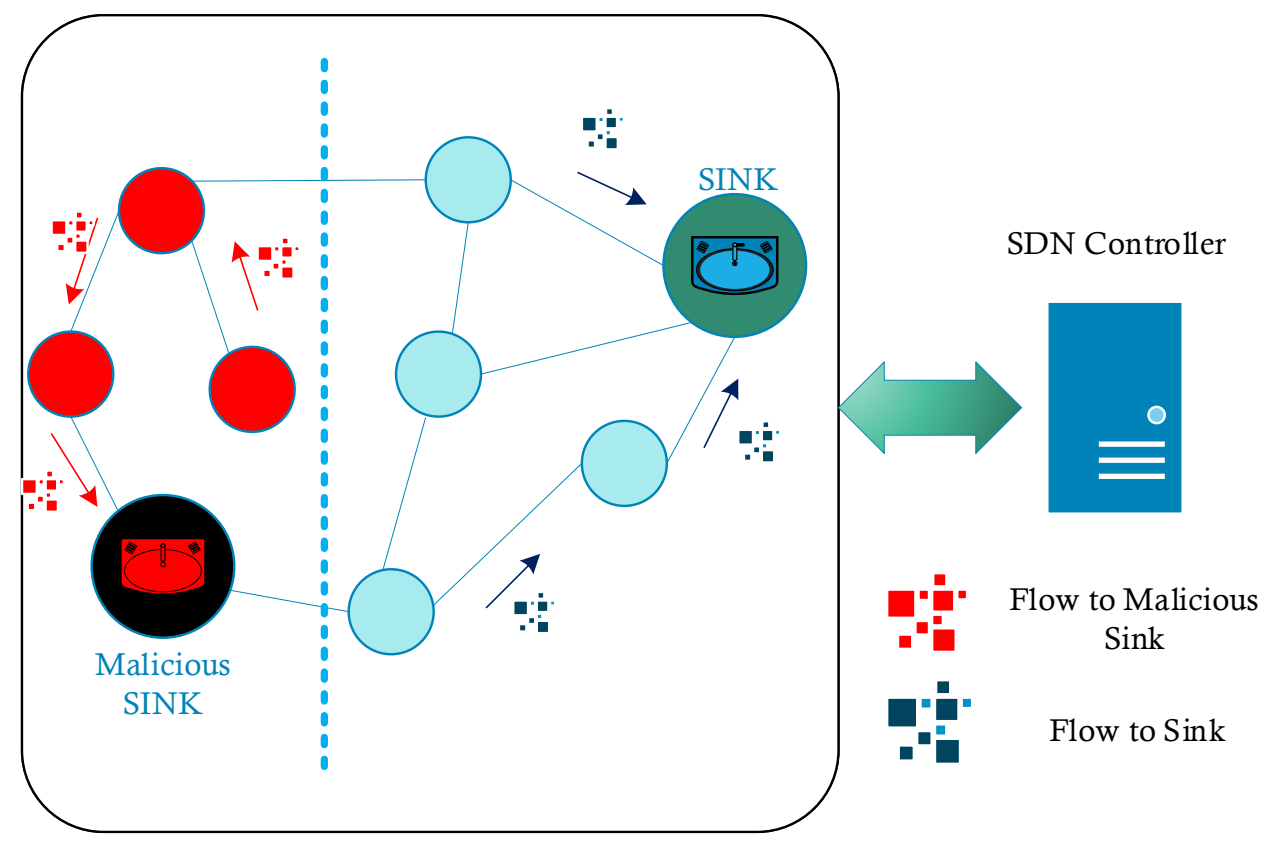

Figure 9: False sink node: a malicious sink can steal application data from nearest nodes by advertising itself as an authentic sink.

\subsection{Chapter Considerations}

Table 3 lists the attacks described in this chapter, summarizing the security aspects they affect and the attack's corresponding consequences.

Table 3: Attacks against IT-SDN: a summary.

\begin{tabular}{|l|l|l|}
\cline { 2 - 3 } \multicolumn{1}{c|}{} & Affected security aspect & Consequence \\
\hline Man-in-the-Middle (active) & Authenticity, Confidentiality, Integrity & DoS, Falsified Data \\
\hline Man-in-the-Middle (passive) & Confidentiality & Fingerprinting, Stolen Data \\
\hline Falsifying Controller Node & Authenticity, Confidentiality & DoS, Stolen Data \\
\hline Falsifying Sink Node & Authenticity, Integrity & Sinkhole, Stolen Data \\
\hline
\end{tabular}

An active MitM attack can lead to packet falsification, impersonation of legitimate nodes and DoS in general if the malicious node decides to drop packets instead 
of forwarding them. Therefore, this simple attack can compromise the architecture's confidentiality, authenticity, and integrity. A passive MitM attack, on the other hand, leads in principle to a lower direct damage to the system, since it only affects data confidentiality. However, since passive attackers are harder to detect (WANG et al., 2016), they can remain in the network for a longer time, mapping its architecture aiming to build a more serious attack later; therefore, the threat of such a low-risk attack cannot be ignored.

As the framework currently is, two or more controllers are not supported, the framework is designed to support only one controller. Even if authenticity and integrity is enforced, it will only solve the problem of stolen data caused by a malicious controller. In the case of a new authorized controller introduced in the network, the new controller will cause a misconfiguration problem, leading to the DoS of one of the controllers. A more robust west/eastbound interface is required, where communication between them is specified to solve this issue.

Falsifying the sink node enables attackers to steal any target application's data, In addition, by learning what kind of applications are installed in the network, attackers can fabricate packets with the required format and forward them to the original sink, disrupting the target application's behavior.

Dealing with such attacks against the IT-SDN framework, while preventing a noticeable performance degradation, is the goal of the solution proposed in the following chapter. 


\section{PROPOSED SOLUTION}

This works has as the main objective the implementation of a security framework in the IT-SDN, thus preventing the attacks presented in Chapter 3. In summary, the proposal provides confidentiality, integrity and authenticity services, which are standard requirements in the context of WSN and IoT (ATZORI; IERA; MORABITO, 2014). Similarly to WS3N, our proposal builds upon iSMQV and security services for enabling the establishment of secure channels among the network nodes. Unlike WS3N, however, in the proposed solution the asymmetric key bootstrap is performed out-ofband, rather than upon the node's arrival at the network. By employing in-band, like in the WS3N, the framework is susceptible to spoofing MAC address attacks (CARDENAS, 2003). A malicious node can falsify the MAC address and request its own implicit certified signed by the KGC. Not only that, our work also enforces an AKA with the Sink node, so that any data sent to it can be authenticate and/or encrypted. We also want to evaluate the framework when the security services are implemented with 128 bits of security level, so we also evaluate the performance with said security level. Lastly, we want our framework to represent a real world deployment scenario as much as possible, to do this we want the security services to be implemented on-demand. In summary the main differences of this work to the WS3N are as follow.

- Out-of-band asymmetric key bootstrap.

- Nodes do the AKA with the Sink, and send authenticated/encrypted data to it

- 128 bits security level evaluation 
- On-Demand security

\subsection{Requirements}

In this sub-section we list relevant requirements for our framework.

- On-Demand Security: Some nodes in the network may handle more sensitive data than others, so their need for authenticity, integrity and confidentiality may differ. Hence, the framework does not require that all nodes have valid keys from the start, which means that any sensor node that supports IT-SDN may be introduced in the network. Security services are then only employed if requested by a node, as long as the communication end-points have valid public-private key pairs.

- Security Levels: Some deployments may require a modern, 128-bit security level, while others may prefer a legacy, 80-bit security for compatibility purposes or to avoid some computational overhead. For this reason, the proposed framework support both 128- and 80-bit security.

- Support for different security services: The network administrator can specify what security services are required for a flow. Three options are available: (0): No security service is required, so the communication avoids unnecessary overhead related to security algorithms; (1): Authentication-only, meaning that the packets' authenticity and integrity is ensured during the communication using a message authentication code (MAC) (SIMPLICIO et al., 2013a); (2): Authenticated-encryption, in which case an authenticated-encryption with associated data (AEAD) scheme is employed to provide data confidentiality besides authenticity and integrity (SIMPLICIO et al., 2013a).

- End-to-End Security: Nodes support end-to-end secure communications between the controller and sensor nodes, as well as between sink and sensor nodes. 


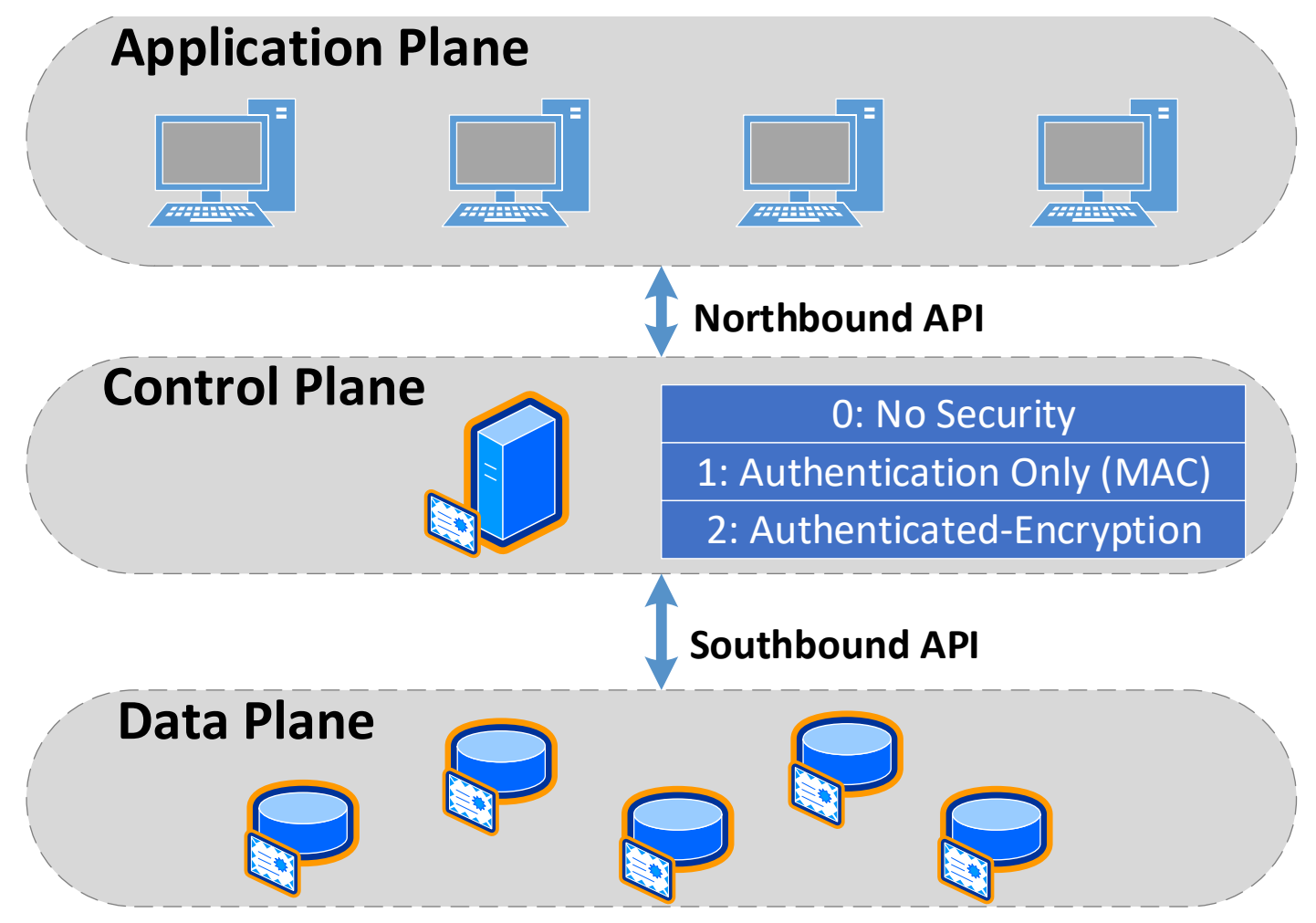

Figure 10: Operation modes available for the control and application planes; no security, where no security services are employed; authentication only, a MAC is used to guarantee authenticity and integrity; authenticated-encryption, AEAD scheme is employed to add confidentiality.

To enforce the above characteristics the control plane is responsible to instruct the security services it wants in the communication between itself and sensor nodes. While the application plane is responsible to instruct the security services in the communication between the sensor and sink nodes. There are currently three levels of security as shown at Figure 10.

\subsection{Building Blocks}

iSMQV (SIMPLICIO JR et al., 2017) is the AKA solution chosen for the bootstrap of the asymmetric keys and the exchange of symmetric keys in the proposed solution. Two flag bits are used to indicate the enforcement of security operations, namely confidentiality and integrity+authenticity, so each of such security services are made 
optional. The proposed solution performs the AKA protocol through iSMQV, whereas authenticated encryption procedures rely on the LetterSoup scheme (SIMPLICIO JR. et al., 2009). The choice of such algorithms is motivated in the following sub-sections.

\subsection{1 iSMQV}

As briefly mentioned in Section 2.2.2, iSMQV is an authenticated key agreement (AKA) that combines SMQV (SARR; ELBAZ-VINCENT; BAJARD, 2010) with implicit certificates (BROWN; GALLANT; VANSTONE, 2002; CAMPAGNA, 2008). The implicit certification model contrasts with conventional because, in the latter the public key is explicitly listed together with its owner's identification data and the digital signature created by the certificate authority (CA); hence, the public key and digital signature can be seen as distinct, unrelated information. Conversely, in implicit certificates the public key is reconstructed from the data set that comprises the implicit certificate: the ID of its owner, and a credential created with the help of a Key Generation Center (no explicit signature exists). This reduces the amount of data required for the representation of implicit certificates when compared with conventional ones. Then, when verifying data signed with implicit certificates, the validity of the public key is indirectly ascertained by the validity of the signature on the data itself.

iSMQV requires only conventional elliptic curve point operations (MILLER, 1985; KOBLITZ, 1987) (e.g., it does not rely on no bilinear pairing, which are usually computationally expensive (SAKAI; OHGISHI; KASAHARA, 2000)). It is also escrow-less, meaning that no entity other than the owner of a private key knows its value. With the help of the Key Generation Center, the nodes are able to bootstrap public/private key pairs $\left(K_{p u b}, K_{p r i v}\right)$, and their corresponding implicit certificates. By utilizing this implicit public key validation, any pair of nodes can then run an elliptic-curve version of SMQV for establishing symmetric keys among themselves, as depicted in Figure 11. 


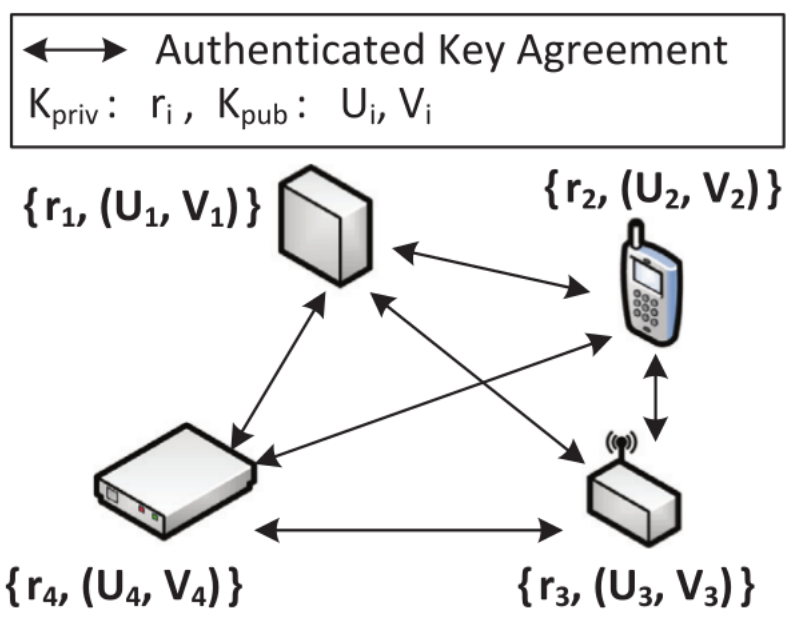

Figure 11: iSMQV in the IoT scenario: $r_{i}$ is the private key and the set $\left(U_{i}, V_{i}\right)$ is the public key; each node sends its public key along with its ID, and both parties can check if such key were indeed authorized by the KGC, and then compute a shared key with this information. (SIMPLICIO JR et al., 2017)

Considering the resource-constrained devices present in SDWSN, iSMQV is relevant in this scenario due to its lightweight properties. The protocol is then employed assuming an out-of-band verification of the nodes' authenticity, so only valid nodes are authorized to get valid credentials from the KGC (bootstrap phase) by the network's administrators.

\subsubsection{Authenticated Encryption with Associated Data}

Authenticated Encryption with Associated Data (AEAD) schemes are symmetric key mechanisms that provide confidentiality, integrity and authenticity with a single secret key. It provides confidentiality through the encryption of the message and authentication and integrity by computing the MAC of the message. In some cases, the operation of these schemes allows to authenticate the entirety of a message while encrypting only part of it. This is desirable in our scenario because of two aspects: (1) we do not want the header to be encrypted because of the routing algorithm of the IT-SDN; (2) we want to use only one symmetric key. After all, if authentication and encryption is employed with different algorithms for each process (e.g., HMAC and AES), then it is usually considered a good practice to compute and use two distinct symmetric keys, 
one for HMAC and another for AES.

The choice of LetterSoup(SIMPLICIO JR. et al., 2009) as the AEAD scheme adopted in the architecture, on the other hand, was motivated by (1) the fact that it display good performance in this scenario when compared with other, more conventional schemes (SIMPLICIO et al., 2013b), and (2) the prompt availability of the algorithm for our testbed platforms (MARGI et al., 2010). Other potential choices for this scenario would be the Ascon (V1.2, 2016) and ACORN ((V3), 2016) schemes, the AEAD schemes that won

the recently finished CAESAR competition (https://competitions.cr.yp.to/ caesar-submissions.html), in the "resource constrained environments" category. However, evaluating such alternatives is left as a topic for future work.

\subsection{Secure IT-SDN}

In this section, we describe how the Secure IT-SDN model works from the bootstrap of the network, until the full bootstrap of the security services.

\subsubsection{Public/Private Key Bootstrap}

The first step for any node that needs to engage in secure communications is to obtain a valid public-private key pair with an authorized Key Generation Center (KGC). In the proposed framework, we assume that this procedure is performed by the network administrator, in an out-of-band manner, using the iSMQV protocol (SIMPLICIO JR et al., 2017). As a result, the node obtains a private key $r$ and a corresponding public key $Y$ that is implicitly certified by the KGC (CERTICOM, 2013). The adoption of implicit certification reduces the amount of information that needs to be exchanged by nodes when verifying the authenticity of each other's public keys. Namely, implicitly certified public keys consist of two elliptic curve points (HANKERSON; MENEZES; VANSTONE, 2003), which translate roughly to $4 k$ bits of information for a system whose 


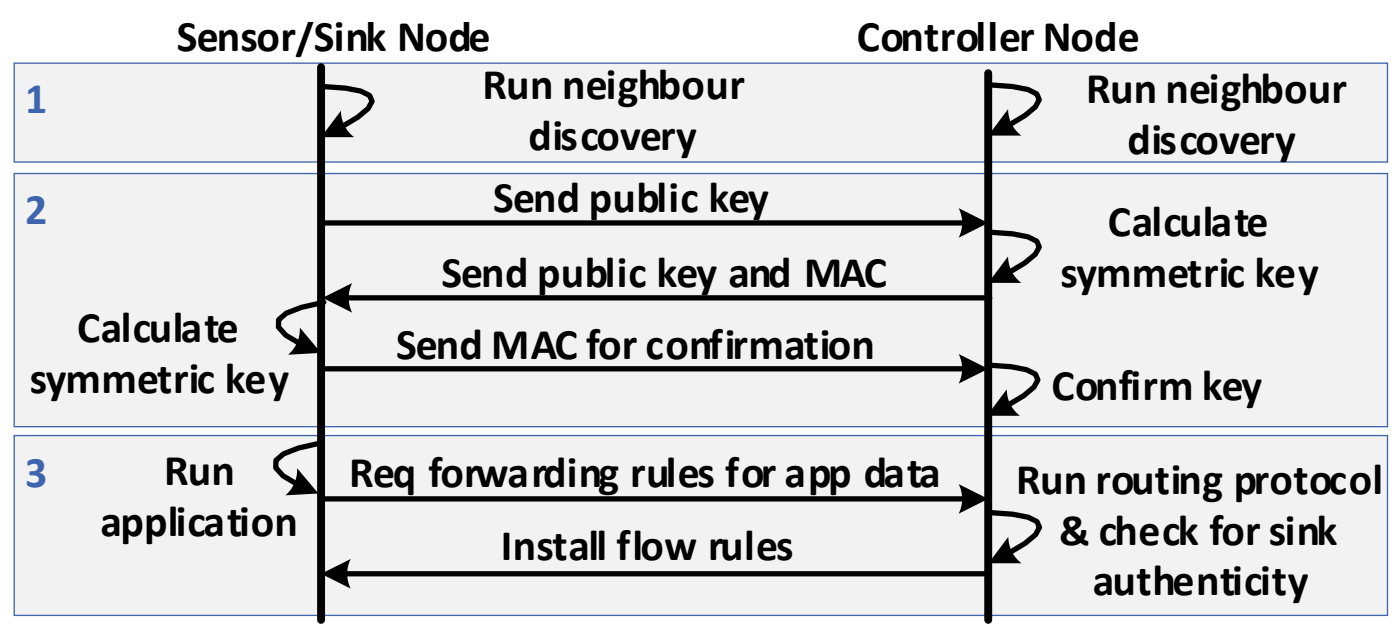

Figure 12: The key establishment process with the controller; sensor/sink nodes start the process; after both keys are confirmed the sensor node runs its application and request flow rules to the controller.

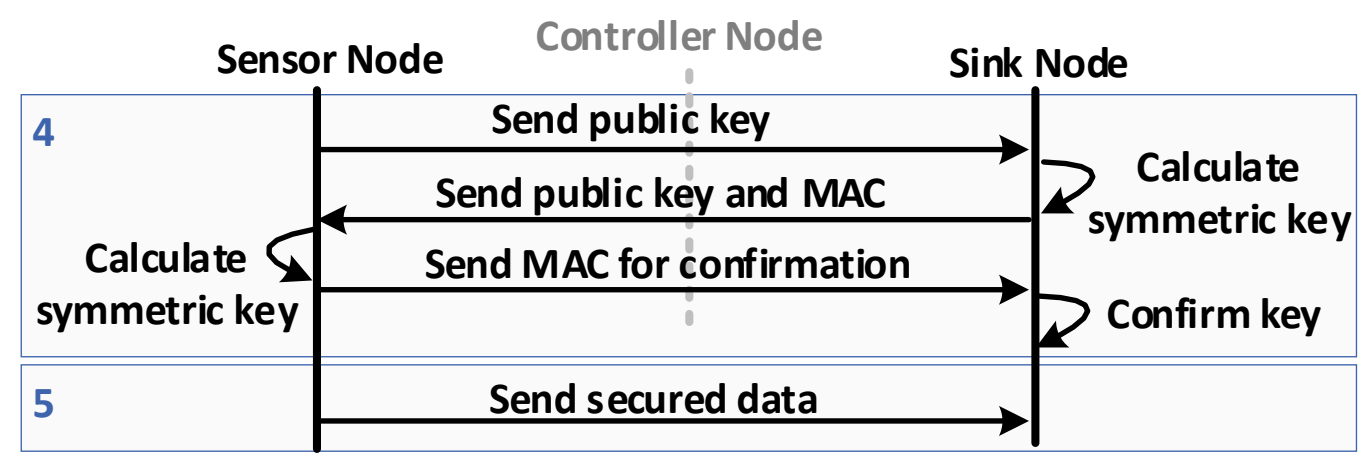

Figure 13: The key establishment between sensor and sink nodes; after the sensor node requests the flow rules to the controller, the sensor node starts the AKA process with the sink; finally, after calculating the symmetric key, the sensor node can send encrypted/authenticated data.

security level is $k$ bits.

\subsubsection{Symmetric Key Establishment}

After entering the network, nodes that desire to use security services must establish a secure channel with the controller and with relevant sinks. This procedure is depicted in Figures 12 and 13, and can be summarized as follows:

1. First, sensors and sink nodes run the neighbour discovery protocol, learning 
about other nodes in the network and get a valid route to the controller.

2. All interested nodes then run the iSMQV protocol with the controller, aiming to establish a secure channel with it. This procedure requires nodes to inform their own public keys to the controller, which is combined with the controller's private key to generate a secret, symmetric key. The controller then responds with its own public key to the requesting node. Also, to confirm that a valid symmetric key was calculated, the controller authenticates this response packet with the symmetric key, using a MAC scheme. Finally, the sensor/sink receives generate the same key and sends an authenticated confirmation to the controller, proving that the protocol was correctly executed.

3. Subsequently, the sensor nodes can run applications and send data flow requests to the controller. If the application packet requires some security service, this need is also informed in the flow request. Unsecured requests are handled as in IT-SDN, by finding an appropriate sink and installing the required forwarding rules in the network. If some security service is required, though, the list of candidate sinks is restricted to authenticated nodes, i.e., sinks that have already established a secure channel with the controller; if no suitable candidate is found, an error message is sent to the requesting node.

4. When an authenticated sink is identified, the controller acts as intermediary for enabling the key establishment between that sink and the requesting sensor node. The result is that an end-to-end secure channel is established between those nodes.

5. Finally, the application plane can enforce the desired security services and send the data to the sink. 


\begin{tabular}{|c|c|c|c|c|c|c|c|}
\hline 2 bits & 6 bits & 8 bits & 8 bits & 8 bits & 16 bits & Varies & 64 bits \\
\hline Security Flags & $\begin{array}{l}\text { Type of } \\
\text { packet }\end{array}$ & Reserved & Time to Live & $\begin{array}{l}\text { Sequence } \\
\text { Number }\end{array}$ & $\begin{array}{c}\text { Source } \\
\text { Address }\end{array}$ & $\begin{array}{c}\text { Application } \\
\text { Data }\end{array}$ & MAC \\
\hline
\end{tabular}

Figure 14: Packet header employed in the proposed security-enabled SDWSN approach: a 2-bit security flags field allows nodes to indicate the security services to be enforced for the packet.

\subsubsection{Message format}

To enforce the supported security services, we use 2 bits on the header of the IT-SDN packets, as depicted in Figure 14. When a node receives messages in the system, it analyzes the security flags and take one of the following actions: $(\mathbb{\theta})$ process the packet normally without any security services; (1) calculate the packet MAC to check its authenticity; (2) employ AEAD to check for its authenticity and decrypt the ciphertext.

Besides using such flag bits, the main modification made to the IT-SDN packets is the addition of a 64-bits MAC field for authentication, and the insertion of a nonce alongside the application data whenever an AEAD scheme is employed.

\subsubsection{0 bits vs 128 bits}

The main difference between 128 bits implementation to the 80 bits is the quantity of packets required for the symmetric key bootstrap. This is due to the size of packets being limited to 127 bytes by the IEEE 802.15.4 standard as specified on Chapter 3 . Figures 15 and 16 show the process to establish a symmetric key. 
Initializer

Responder

\begin{tabular}{|c|c|c|}
\hline send_AKA_request() & & \\
\hline \multirow{5}{*}{$\begin{array}{l}\text { U(x) (21 Bytes) } \\
\text { V(x) (21 Bytes) } \\
\text { Ephemeral(x) (21 Bytes) } \\
\text { Node_Id (up to } 20 \text { Bytes) } \\
\text { Other fields (14 Bytes) }\end{array}$} & & \\
\hline & & treat_AKA_request() \\
\hline & \multirow{10}{*}{ Source Routed Packet } & Calculate Symmetric Key \\
\hline & & \\
\hline & & \\
\hline \multirow{4}{*}{$\begin{array}{l}\text { treat_AKA_reply() } \\
\text { Calculate Symmetric Key } \\
\text { Validate Packet/Key }\end{array}$} & & send_AKA_reply() \\
\hline & & \multirow{4}{*}{$\begin{array}{l}\text { U(y) (21 Bytes) } \\
\text { V(y) (21 Bytes) } \\
\text { Ephemeral(y) (21 Bytes) } \\
\text { Node_ld (up to } 20 \text { Bytes) } \\
\text { Other fields (14+ Bytes, } \\
\text { MAC included) }\end{array}$} \\
\hline & & \\
\hline & & \\
\hline & & \\
\hline send_AKA_challenge() & & \\
\hline \multirow{2}{*}{$\begin{array}{l}\text { Other fields (14 Bytes, } \\
\text { Mac inluded) }\end{array}$} & & treat_AKA_challenge() \\
\hline & & Validade key/packet \\
\hline
\end{tabular}

Figure 15: The internal flowchart of the AKA algorithm for 80 bits security level in the IT-SDN. The size of each packet must respect the 127 bytes limitation.

$\mathrm{V}(\mathrm{x})$ and $\mathrm{U}(\mathrm{x})$ are the public key parameters previously bootstrapped by the KGC. Ephemeral is a public key generated every time a node wants to do the AKA, it is used during the iSMQV protocol. Other fields represent the rest of the packet, while node id is the node's identifier (e.g., its MAC address). For the 80-bits security level, there are only 3 messages required to complete the AKA; in comparison, for 128 bits there is a total of 5 messages. Another difference is that, for both security levels, the nodes need to store the public key on its memory until the end of the process. Both of these aspects may negatively impact the network: the higher number of messages increases the possibility of packet collisions, whereas the larger memory usage may lead to memory overflows more easily (e.g. if a node receives more than one AKA 
Initializer

Responder

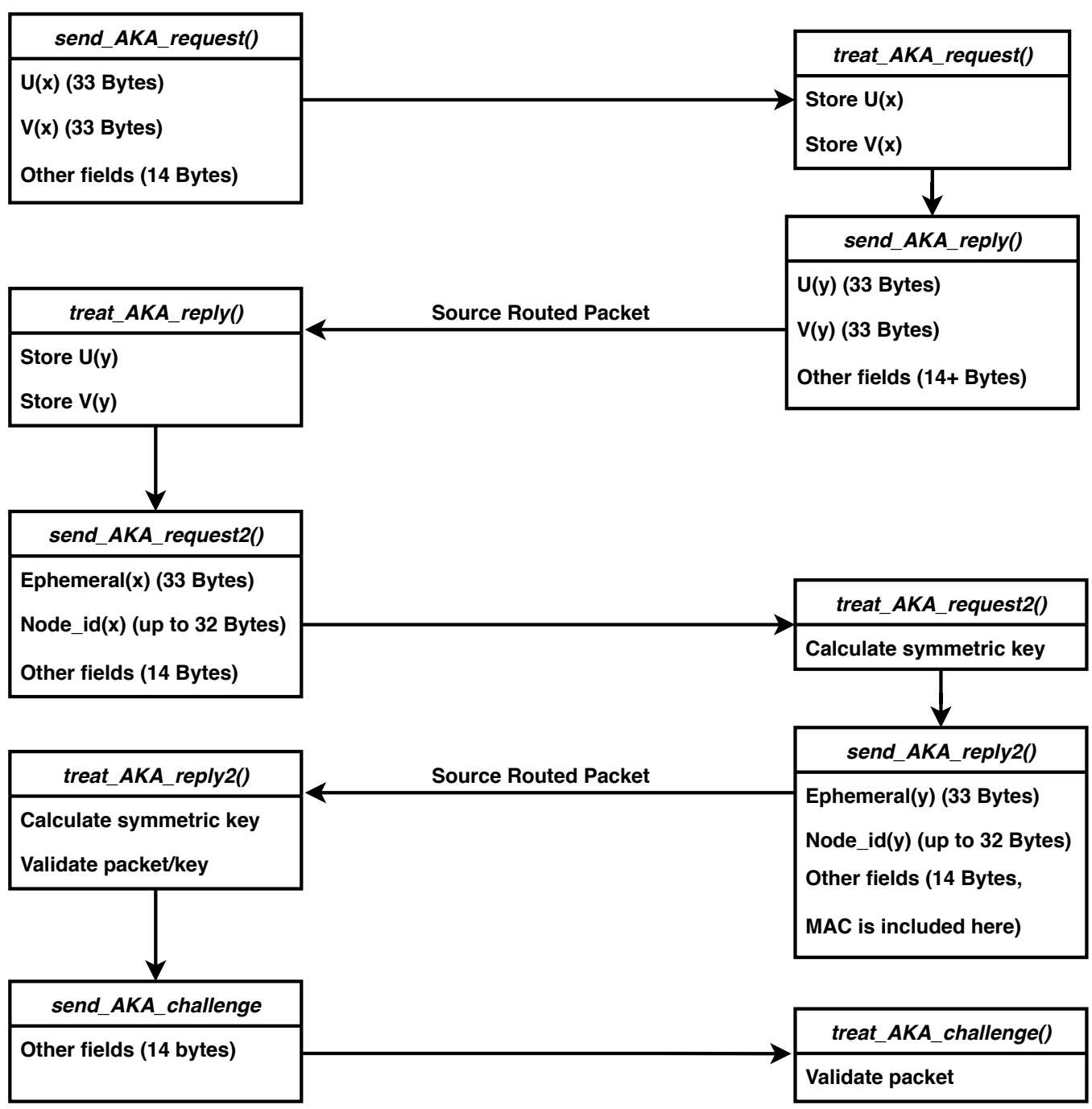

Figure 16: The internal flowchart of the AKA algorithm for 128 bits security level in the IT-SDN. The 128 bits algorithm has two additional messages to respect the 127 bytes limitation.

request message and tries to process them all, it would store the public key until the process complete).

\subsubsection{Mitigating Man-in-the-Middle attacks}

Mitigation of the MitM attack involves encrypting and authenticating packets with an AEAD scheme after the symmetric key agreement, as depicted in Figure 17. 


\begin{tabular}{|c|c|c|c|c|c|c|c|c|}
\hline 2 bits & 6 bits & 8 bits & 8 bits & 8 bits & 16 bits & Varies & Varies & 64 bits \\
\hline $\begin{array}{l}\text { Security } \\
\text { Flags }\end{array}$ & $\begin{array}{l}\text { Type of } \\
\text { packet }\end{array}$ & Reserved & $\begin{array}{l}\text { Time to } \\
\text { Live }\end{array}$ & $\begin{array}{c}\text { Sequence } \\
\text { Number }\end{array}$ & $\begin{array}{c}\text { Source } \\
\text { Address }\end{array}$ & $\begin{array}{c}\text { Application } \\
\text { Data }\end{array}$ & Nonce & MAC \\
\hline
\end{tabular}

Figure 17: The header after being encrypted and authenticated, the security flags are set to 11 (in binary), so the application data is encrypted and a nonce is allocated.

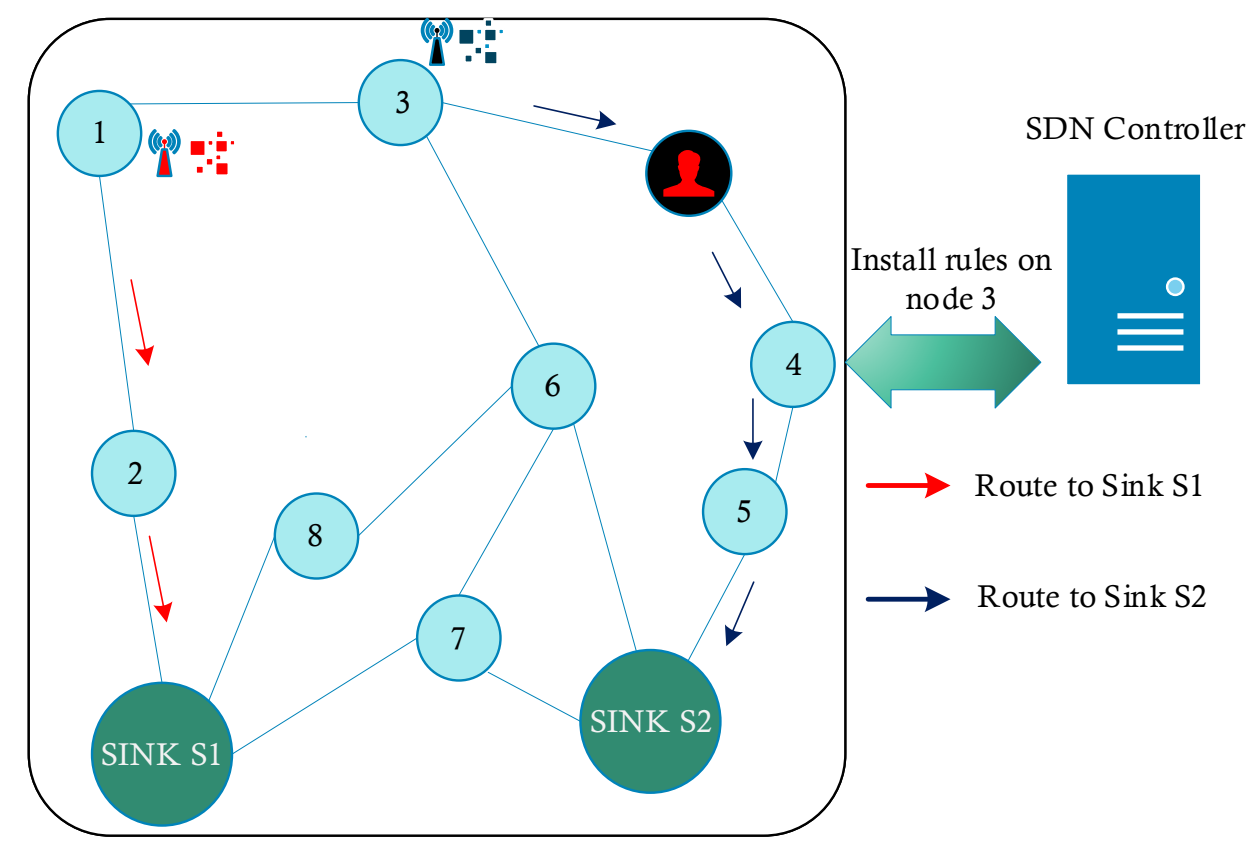

Figure 18: Man-in-the-middle mitigation: Node 3 is configured to encrypt and authenticate its applications packets before sending them; attackers are unable to eavesdrop or modify packets.

We exemplify in Figure 18 how this approach can mitigate MitM attacks. In this scenario, the intruder is located between node 3 and sink S2. Node 3 is unaware of the presence of the intruder next to it, but this legitimate node is configured to send messages only in the Authenticated-Encryption mode. Hence, after confirming the sink's authenticity through the key establishment process, and employing AEAD primitives in the packet, the source node (in this case, node 3) forwards the packet to the attacker. The attacker can then take a few actions. First, the attacker could try 
to modify the data in the packet; however, when any modified packets arrive at the sink, they are discarded after its authenticity is verified. The attacker is also unable to eavesdrop application data, as it is encrypted with the secret key established between the sink and the node.

\subsubsection{Mitigating Malicious Controller attack}

When the network starts, the sensor nodes send their neighbor discovery protocol messages and wait for flow rule messages from the controller. After running the neighbor discovery protocol, the key establishment process begins as specified before. Authorized sensor nodes in this secure framework have ground rules to refuse connection with unauthorized controllers. So, if a controller node has not performed the key bootstrap with the KGC beforehand, the sensor node identifies this issue during the key establishment process, rejecting any message from this controller. By enforcing this type of rule, only malicious controllers that have gone through the key bootstrap with the KGC will be able to tamper with the network. But since the key bootstrap is only done by network administrators, it is very unlikely that a malicious controller is able to obtain valid keys from the KGC. This protection also applies to a multiple controller scenario, guaranteeing that a node does not accept requests from any unauthorized controller. Figure 19 illustrates the process to mitigate a malicious controller.

At first sight, this approach may seem to infringe one key aspects of the SDN paradigm, in which nodes from the data plane should not have any level of autonomy, and all rules are left to the controller. Nevertheless, this autonomy only occurs during bootstrap, aiming to enable nodes to identify possible attackers. After that, the network nodes would still follow the rules established by the (authorized) network controller, as usual in any SDN. 


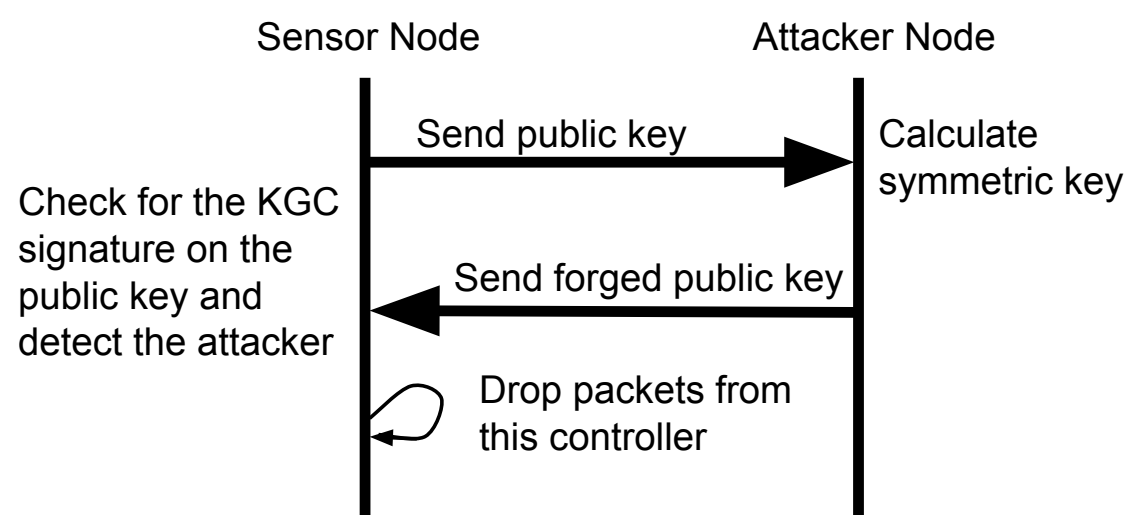

Figure 19: Process to mitigate a falsified controller: sensor node detects attacks when verifying the authenticity of public keys.

\subsubsection{Mitigating Malicious Sink attack}

As discussed in Subsection 4.3.6, it is possible for sensor nodes to detect unauthorized sinks in the network during the key establishment process. However, since we are working with an SDN scenario, sensor nodes must communicate with the controller before sending any information to the sink. The controller should then check the authenticity of potential sinks before installing in sensor nodes any flow rule pointing to that sink, giving an additional layer of protection to the network.

When an authorized node wants to send information with security services enabled, it adds this information into the flow request packet sent to the controller. The controller, after receiving this packet, checks for an authorized sink. Since all nodes do the key establishment with the controller during the bootstrap, the controller knows if a sink node is authorized or not. If an authorized sink is found, the controller installs the required flow rules in the network, and the sensor node starts the key establishment process. However, if the controller does not find any authorized sink, it install flow rules instructing the sensor nodes to drop packets. Figure 20 illustrates this process. 


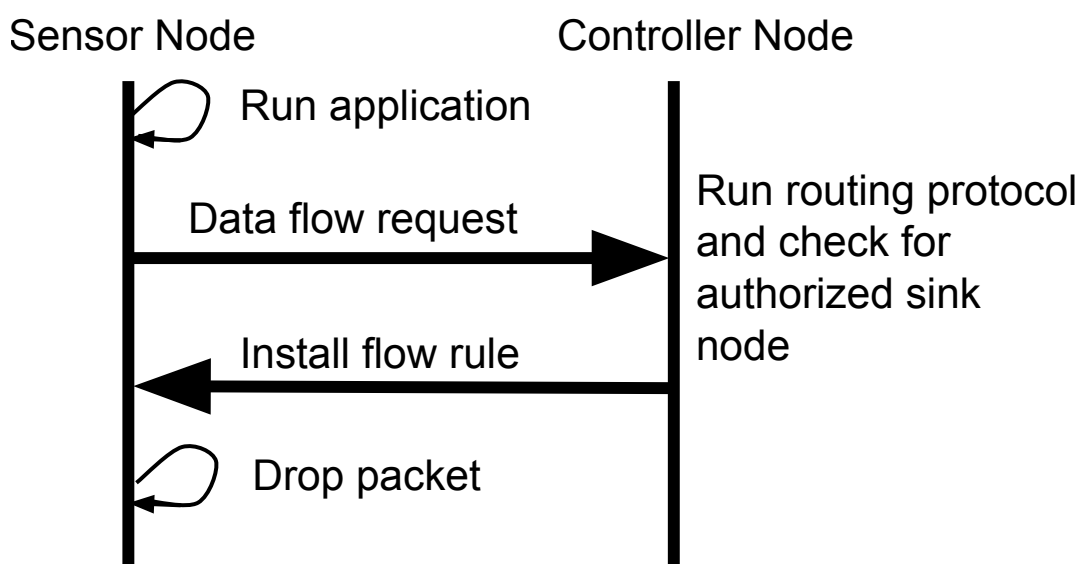

Figure 20: Mitigating a falsified sink node: during a data flow request, the controller checks if both source and sink nodes are authorized; if there is no authorized sink for application, the sensor node is informed to drop the packet.

\subsubsection{Open Issues}

Although the proposal mitigates most of the attacks from 3.1, we emphasize that it is not intended to address all possible attacks against the network. In particular, the solution does not protect against replay attacks (e.g., caused by a MitM), where the intruder re-sends an authentic message from the same node. Hence, if the application's behavior might be subverted by replayed data, it should deploy mechanisms to detect such attacks; typical examples are including nonces or timestamps as part of the payload in each packet. In addition, our proposal does not mitigate fingerprint attacks, where the intruder tries to map the connections of the network using source address fields. Finally, the attacker could drop all application data going to a giving sink, creating a DoS attack. Figure 21 lists the attacks that are mitigated by our solution and some that are not.

Besides such security-related limitations, our proposal inherits one constraint of IT-SDN: the lack of support for multiple controllers. As a result, the same problems discussed in Section 3.1.2, related to the attempt of enforcing two or more (authorized) controllers in the network (resulting in the DoS of the first controller) are not considered. This is one of the main reasons why the security architecture itself only considers 


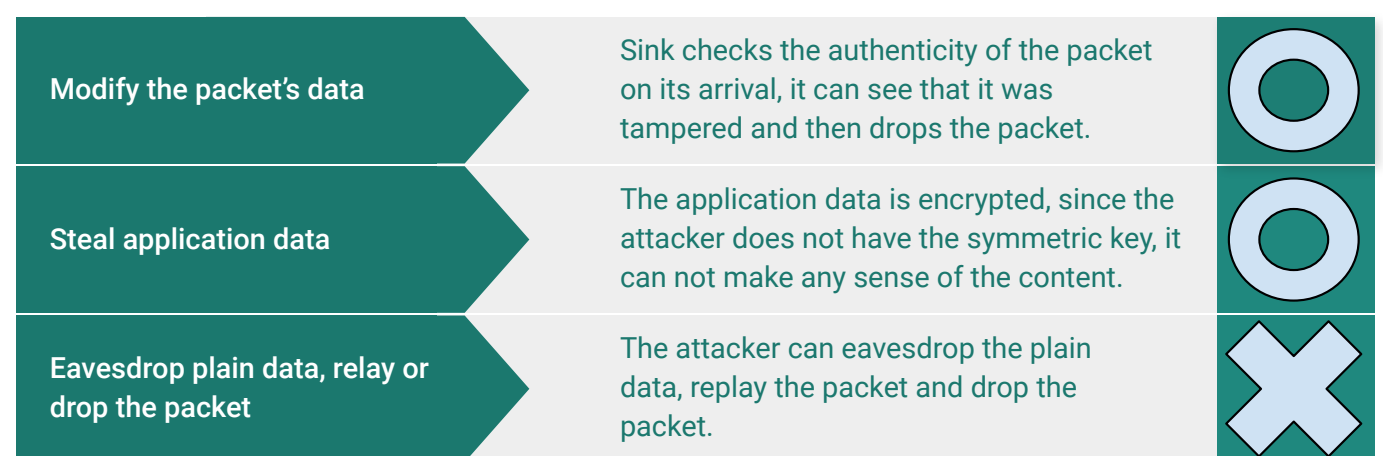

Figure 21: List of attacks mitigated and not mitigated by the proposed security protocols.

one controller. Even though a scalable east/westbound protocol could be employed to solve this issue, so a group of federated controllers could be employed, specifying such solution is considered out of the scope of this work. 


\section{EXPERIMENTAL EVALUATION}

To evaluate the proposed framework, we implement it upon the IT-SDN 0.4.5 and use the Cooja Simulator to extract the results (ROUSSEL; SONG; ZENDRA, 2016). Cooja is a software for simulating WSNs, and can also emulate the execution of binary code on different devices. In this work, we use the Wismote devices as sensor nodes, which are composed of the MSP430 microcontroller (INSTRUMENT, 2010b) (specifically, we use the msp430f5437 chipset (INSTRUMENT, 2010a)) and the CC2520 802.15.4-enabled wireless transceiver (INSTRUMENT, 2010c). Exceptionally, the controller node uses only the wismote to delivery radio messages, whereas all processing is handled in a desktop computer.

IT-SDN 0.4.5 is implemented in C, while the serial communication with the controller is in $\mathrm{C}++$. To enable the cryptography primitives, we use the Relic cryptographic toolkit in its version from June 2019 (ARANHA; GOUVEA, ).

The first result we observe is the run time for each algorithm for a single node. This is shown in Figure 22, considering the average results of 10 simulations. The time it takes for each procedure to complete are considerably lower on a 80-bits security level. Specifically, generating an ephemeral key for the AKA protocol takes 2 seconds, whereas the whole execution of iSMQV takes 9 seconds to complete. In comparison, generating a 128-bits ephemeral key takes 10 seconds and iSMQV runs in 1 minute and 14 seconds. We note that, while the single-core single-thread nodes perform this operation, they remain completely inaccessible, meaning that any packet sent to them 


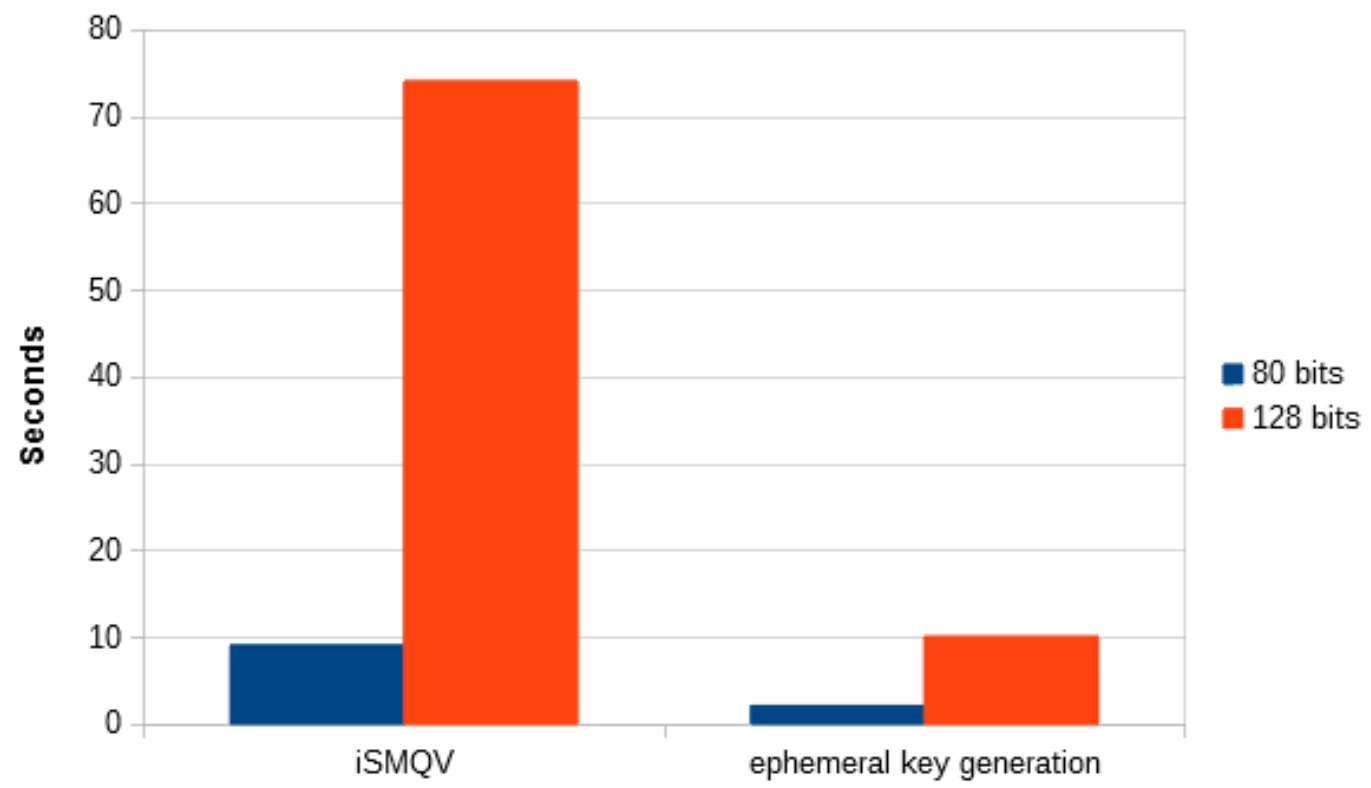

Figure 22: Time for a sensor node to execute the two most expensive procedures in the system: generating ephemeral keys and completing the whole iSMQV protocol.

Table 4: Parameters summary for the simulations with the 80 bits framework without a Sink node.

\begin{tabular}{ll}
\hline Parameter & Value \\
\hline Number of Nodes & $9,25,36,49,64$ \\
Topology & Grid with controller at center \\
Traffic Rate & IT-SDN default (5 seconds per packet) \\
Simulation Time & 20 Minutes \\
Simulation Tests & 10 times $/ 5$ times for 64 nodes \\
\hline
\end{tabular}

is simply dropped. This is not critical for the network operation, though, because this process is not run often; namely, it only occurs during the bootstrap of the network or when a re-keying operation is mandated (e.g., by the network administrator).

Considering the costs of such basic operations, in the next subsections we simulate different scenarios to evaluate the actual impact of the proposed security framework on the whole network. 


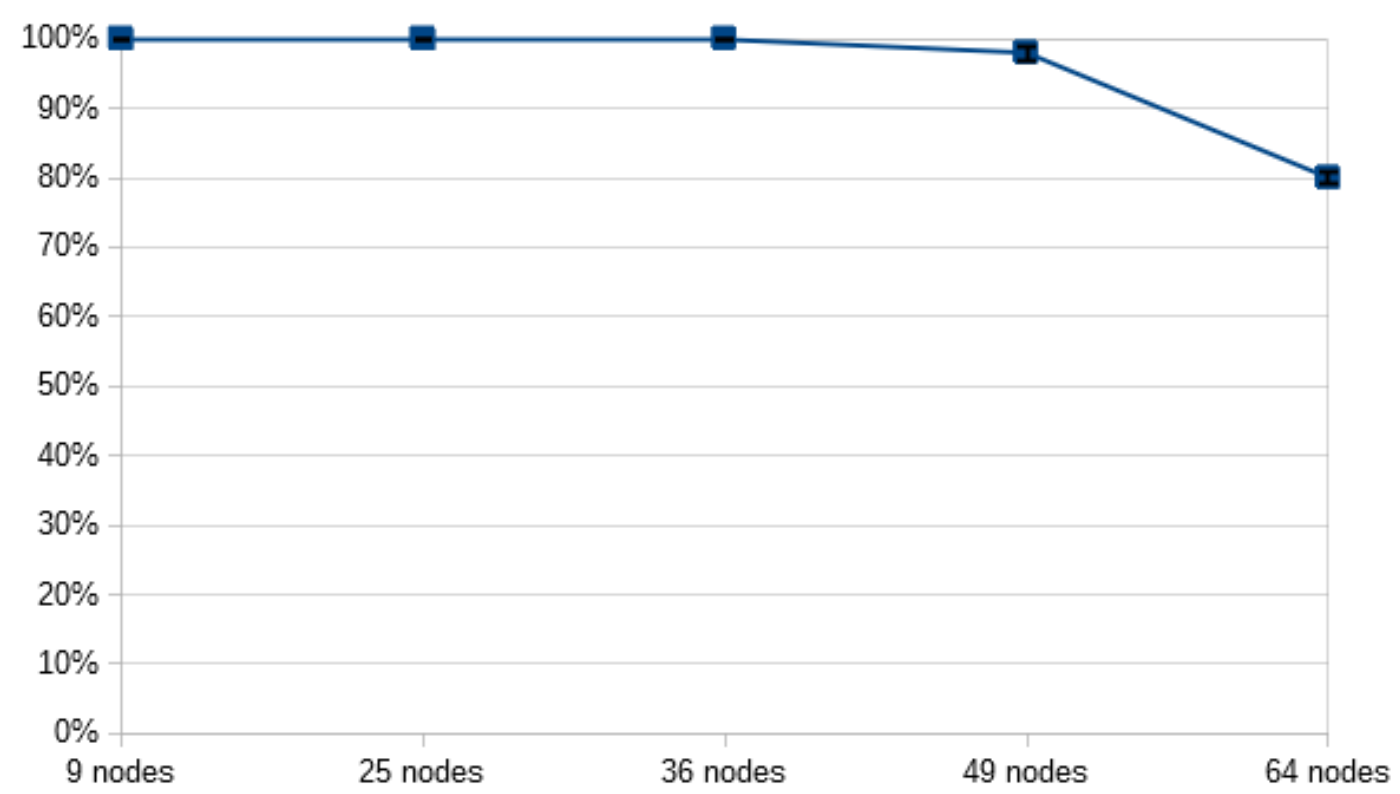

Figure 23: Percentage of nodes that completed the AKA with the controller when running the proposed security architecture with an 80-bits security level.

\subsection{0 bits Security Level Sinkless}

The settings for this scenario are shown in Table 4. Basically, its goal is to evaluate the AKA interactions with the network controller; hence, no sink is included in the network. For this purpose, we run 10 simulations for different network sizes, namely $9,25,36$, and 49 nodes. We also include a scenario with 64 nodes, in which case only 5 simulations are run due to the long execution time and resources consumed in this specific test. The results of these scenarios showed a standard deviation of less than $3.7 \%$.

First, Figure 23 shows the amount of nodes that completed the AKA with the controller within 20 minutes. When analysing the logs from the simulations, we identified that are two main reasons why some nodes could not complete the AKA within this time limit.

1. Packet loss: this is mostly due to packet collisions, which are more common when some nodes are booted simultaneously. 


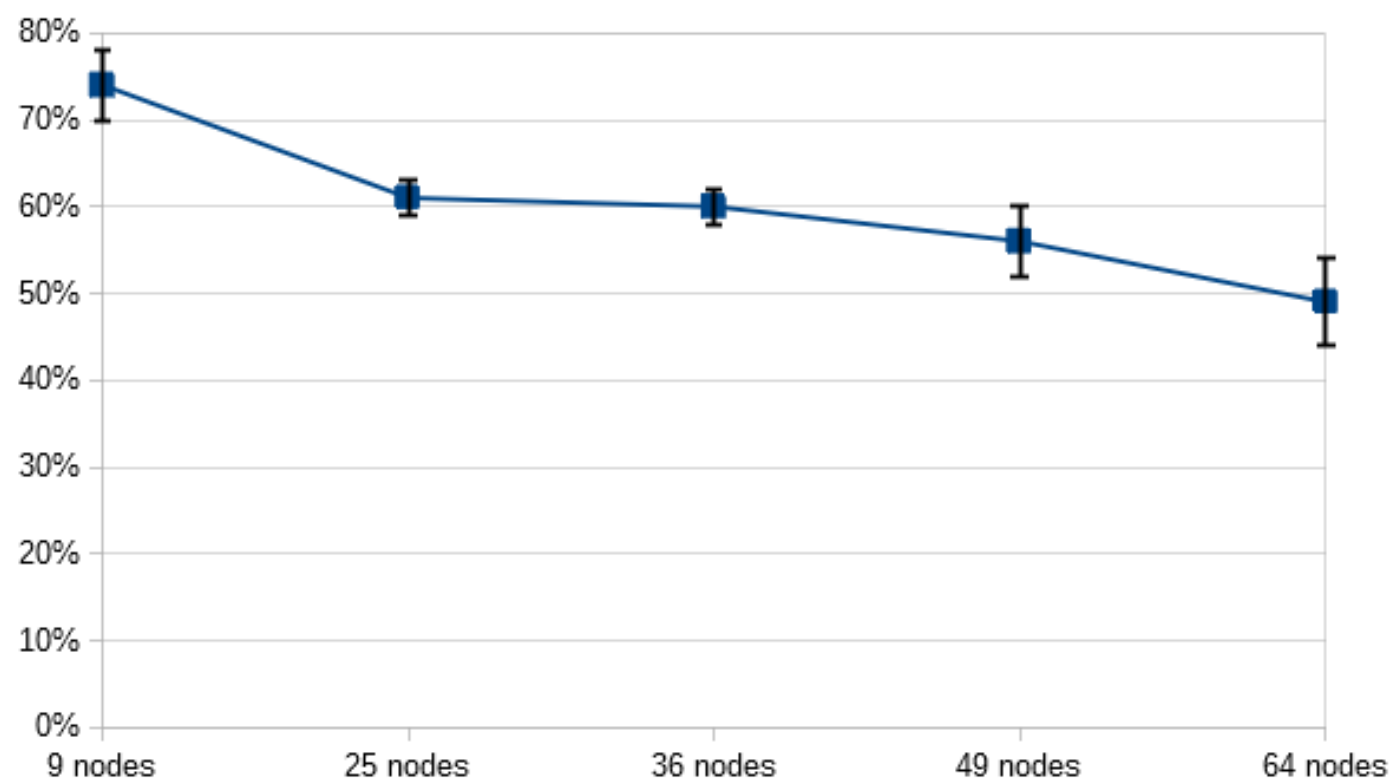

Figure 24: Delivery rate of AKA packets when establishing keys with the controller, for different network sizes. These results are from the 80 bits architecture.

2. Due to the nodes' space limitations, their flow tables could get full quite fast. When an unknown flow arrives at a node, but its flow table is full, it requests new flow rules to the controller, potentially overwriting an older entry. This process consumes a significant amount of time and, when combined with packet collisions, prevents some nodes from completing the AKA in time. As expected, this issue is more common in scenarios comprising more nodes.

In addition, Figure 24 shows the delivery rate of AKA-related packets (request, reply and challenge - as shown in Section 4.3.4) for each network scenario. Again, by analysing the logs of the simulations we could infer two main reasons that justify packet loss: collisions and the IT-SDN reliable transmission protocol. More precisely, if a node sends a packet that requires reliable delivery, but does not receive an acknowledgement after a timeout period, the sender re-transmits the packet. This is a problem by itself in our implementation due to the iSMQV protocol. After all, it takes 9 seconds for the iSMQV protocol to be executed; in a large network, with many hops between each of them, it may be hard to configure an adequate timeout for this proce- 


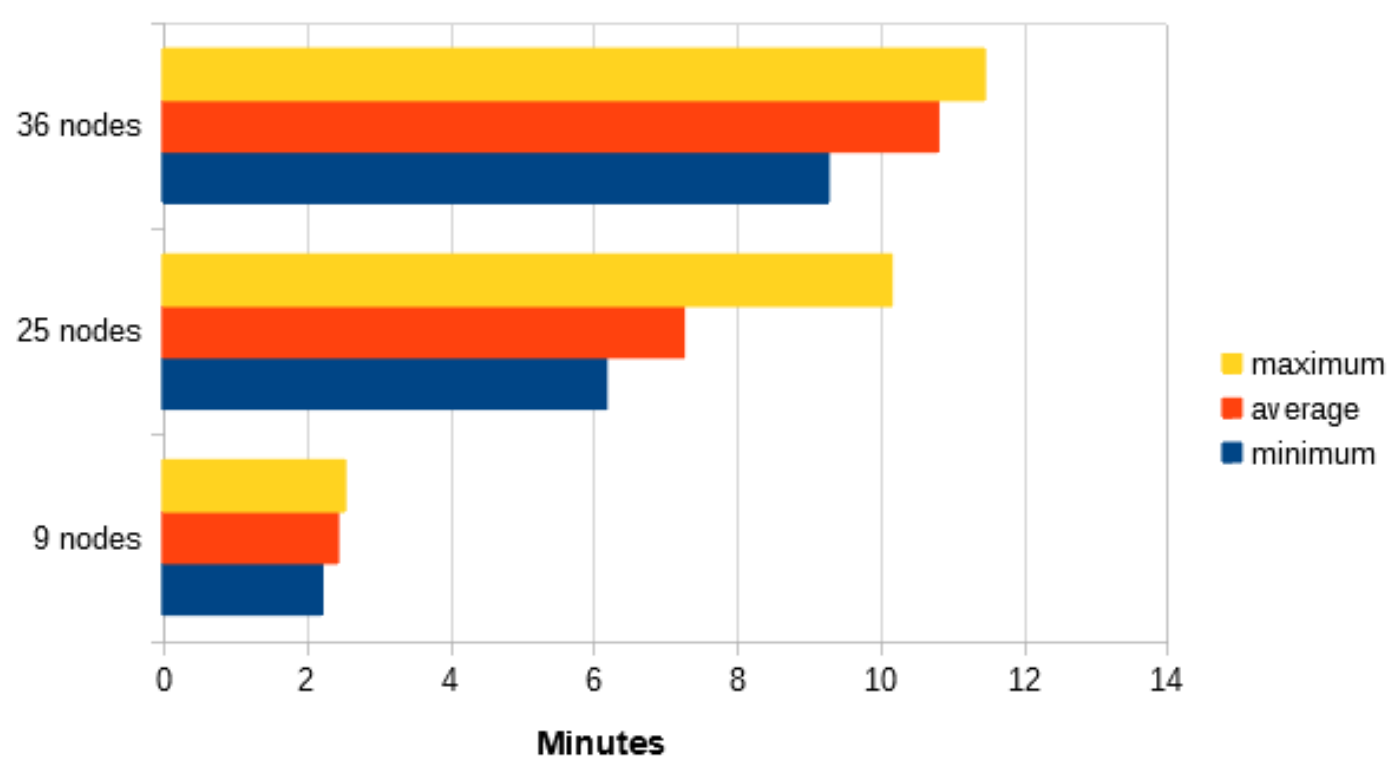

Figure 25: Time taken for the network to perform the AKA with the controller. These results are from the 80 bits architecture. Only the scenarios which completed the AKA during the simulation time are represented.

dure, so packets may be lost and re-transmitted, increasing the network congestion. As a result, the AKA completion rate reaches $100 \%$ only for small network sizes (namely, 9, 25 and 36 nodes). The minimum, average and maximum time taken for all nodes to complete the AKA with the controller is depicted in Figure 25.

\subsection{0 bits Security Level with a Sink}

In a second test, we include a Sink to receive data from nodes. All nodes are configured to send authenticated messages to the sink, meaning that the latter has to perform the AKA with all nodes in the network. The specification of the scenario is detailed in Table 5. Due to memory constraints in the devices, though, the total number of keys stored in each node had to be limited to avoid memory leaks. For this reason, we limited to 20 the maximum number of nodes with which the sink performs the AKA procedure; hence, in scenario comprising additional nodes, only 20 of them are able to communicate simultaneously with a given sink. The results of the scenarios of 
Table 5: Parameters summary for the simulations with an 80-bits security framework and one Sink node.

\begin{tabular}{ll}
\hline Parameter & Value \\
\hline Number of Nodes & $9,25,36$ \\
Topology & Grid with controller and sink at the center \\
Traffic Rate & IT-SDN default (5 seconds per packet) \\
Simulation Time & 20 Minutes \\
Simulation Tests & 5 times \\
\hline
\end{tabular}

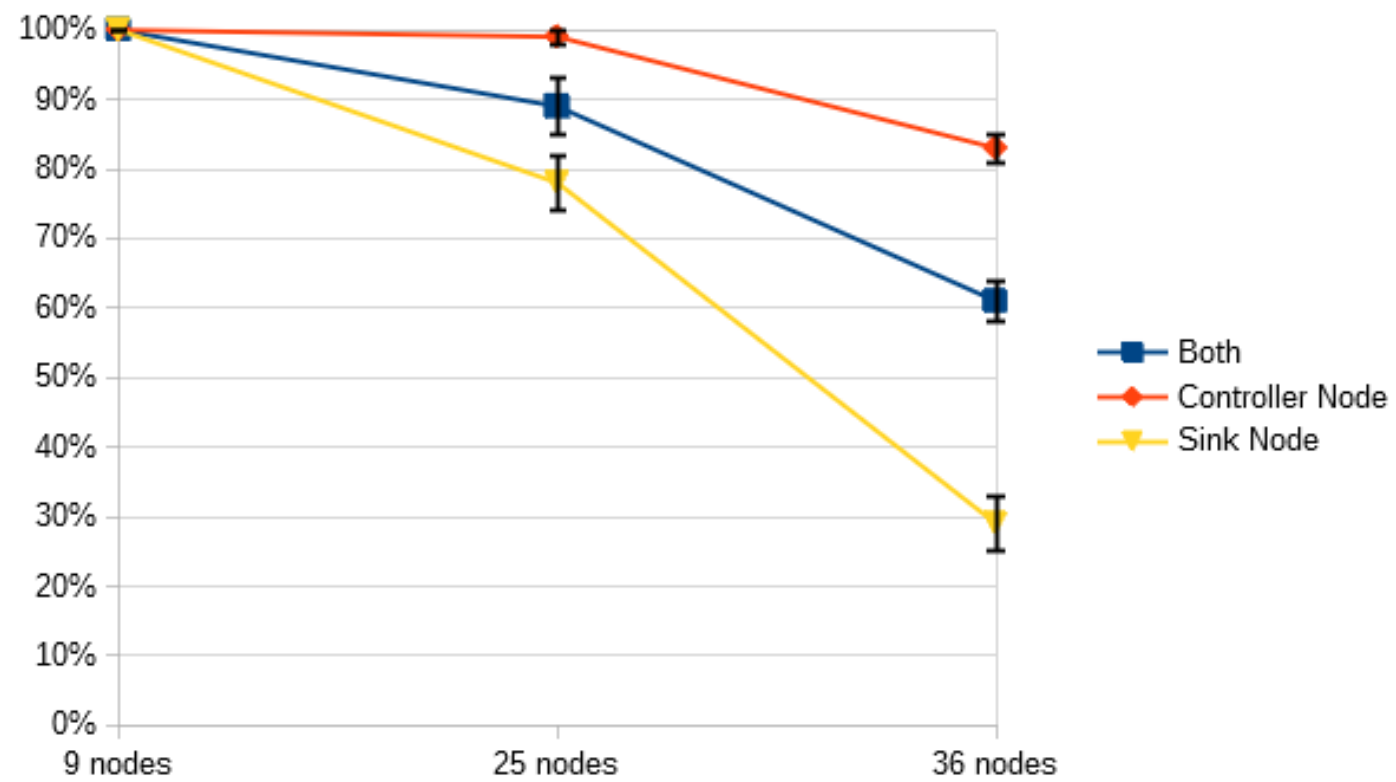

Figure 26: AKA completion rate for the network with a single sink and a single controller, for different numbers of nodes and an 80-bits security level. The curves represent the percentage nodes the controller; sink; and both completed the AKA process.

this section showed a standard deviation of less than $5 \%$.

Figure 26 shows the completion rate of the AKA in the network, considering the procedure between: nodes and controller; nodes and sink; and the overall rate. All values represent the average results obtained from five simulations.

In the scenario comprising 9 nodes, the bootstrap was completed by the whole network. Although the scenario with 25 and 36 nodes has shown a reduced completion rate, most of the nodes still completed the AKA, with nodes located farther away from the controller and sink struggling a little to complete the AKA due to packet loss. 


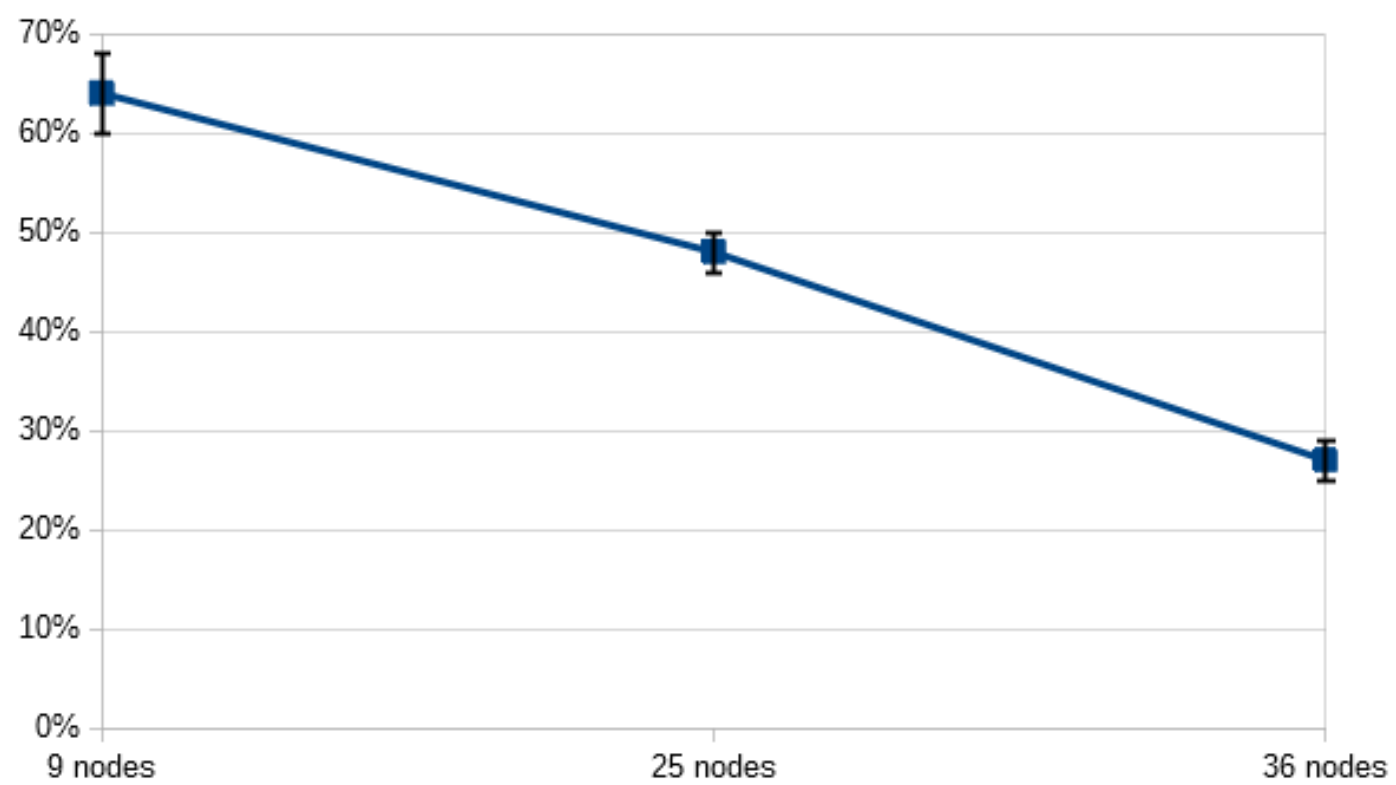

Figure 27: Delivery rate of AKA packets when establishing keys with the controller and sink, for different network sizes. These results are from the 80-bits architecture.

Finally, Figure 27 shows the delivery rate of the AKA packets for each scenario. The decrease in delivery rate when compared to the previous scenario is likely due to the sink node being unable to respond during the AKA process.

\subsection{8 bits Security Level Sinkless}

In this section we repeat the tests performed in Section 5.1 for the 80 bits security level, but for the security level of 128 bits. Table 6 shows the parameters for the simulations without the sink node, considering only scenario where the running time is not excessive (namely, 9, 25, 36 and 49 nodes). The standard deviation is of less than $8 \%$ for the results of this section.

Figure 28 shows the completion rate of the AKA protocol, while Figure 29 shows the delivery rate of AKA packets.

Due to the higher security level, and the consequently higher runtime of iSMQV, we observe a reduced number of completions for the AKA procedure, as well as a 
Table 6: Parameters summary for the simulations with the 128 bits framework without a Sink node.

\begin{tabular}{ll}
\hline Parameter & Value \\
\hline Number of Nodes & $9,25,36,49$ \\
Topology & Grid with controller at center \\
Traffic Rate & IT-SDN default (5 seconds per packet) \\
Simulation Time & 20 Minutes \\
Simulation Tests & 5 \\
\hline
\end{tabular}

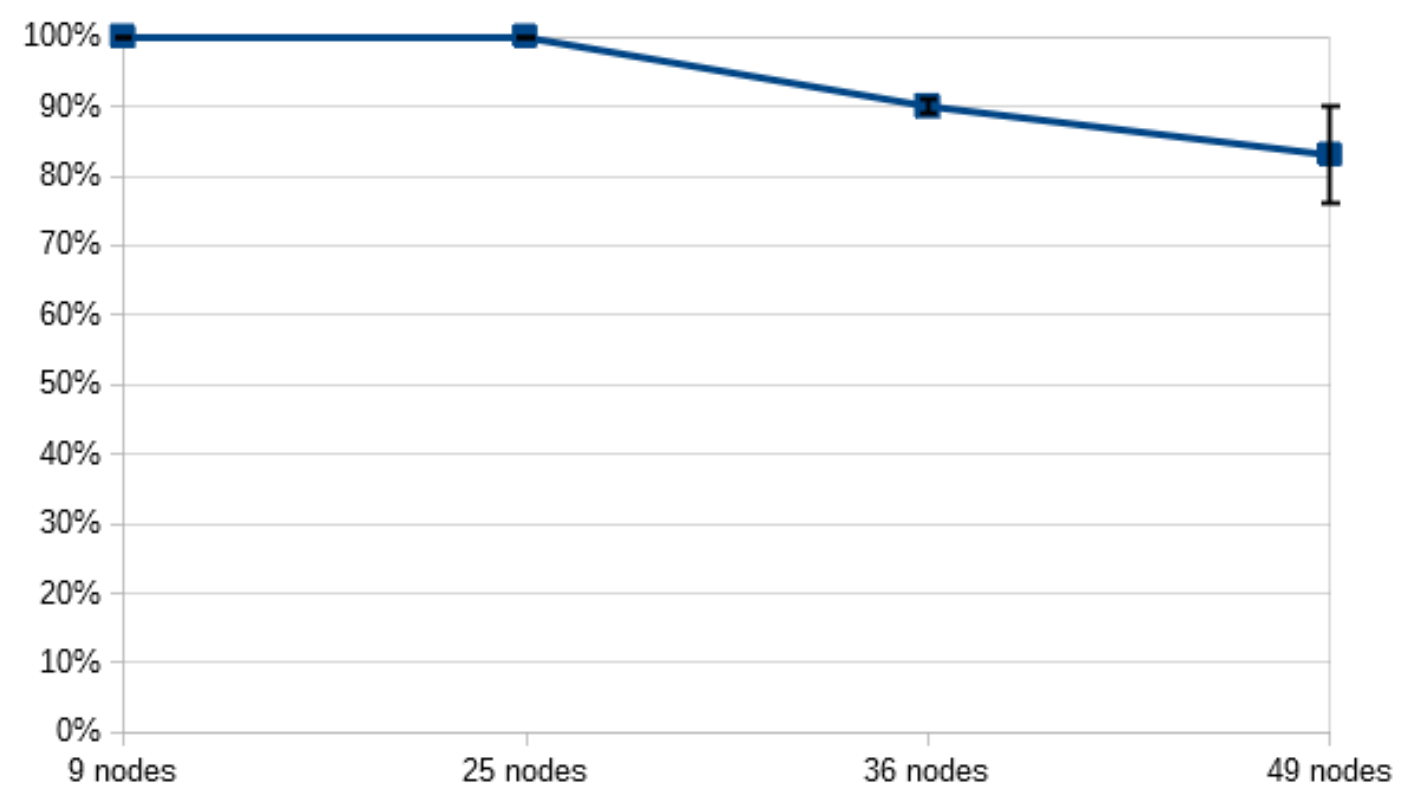

Figure 28: Percentage of nodes that completed the AKA with the controller when running the proposed security architecture with an 128-bits security level.

lower packet delivery rate. Figure 30 shows the time it took for the complete network bootstrap in the scenarios where this was possible (namely, when the network contained 9 and 25 nodes). The numbers obtained correspond roughly to $2 \times$ the time taken by the 80 bits security level.

\subsection{8 bits Security Level with a Sink}

The last group of simulations we run, are the scenarios from Section 5.2. The parameters are represented on Table 7. Just like the previous simulation, we reserve 


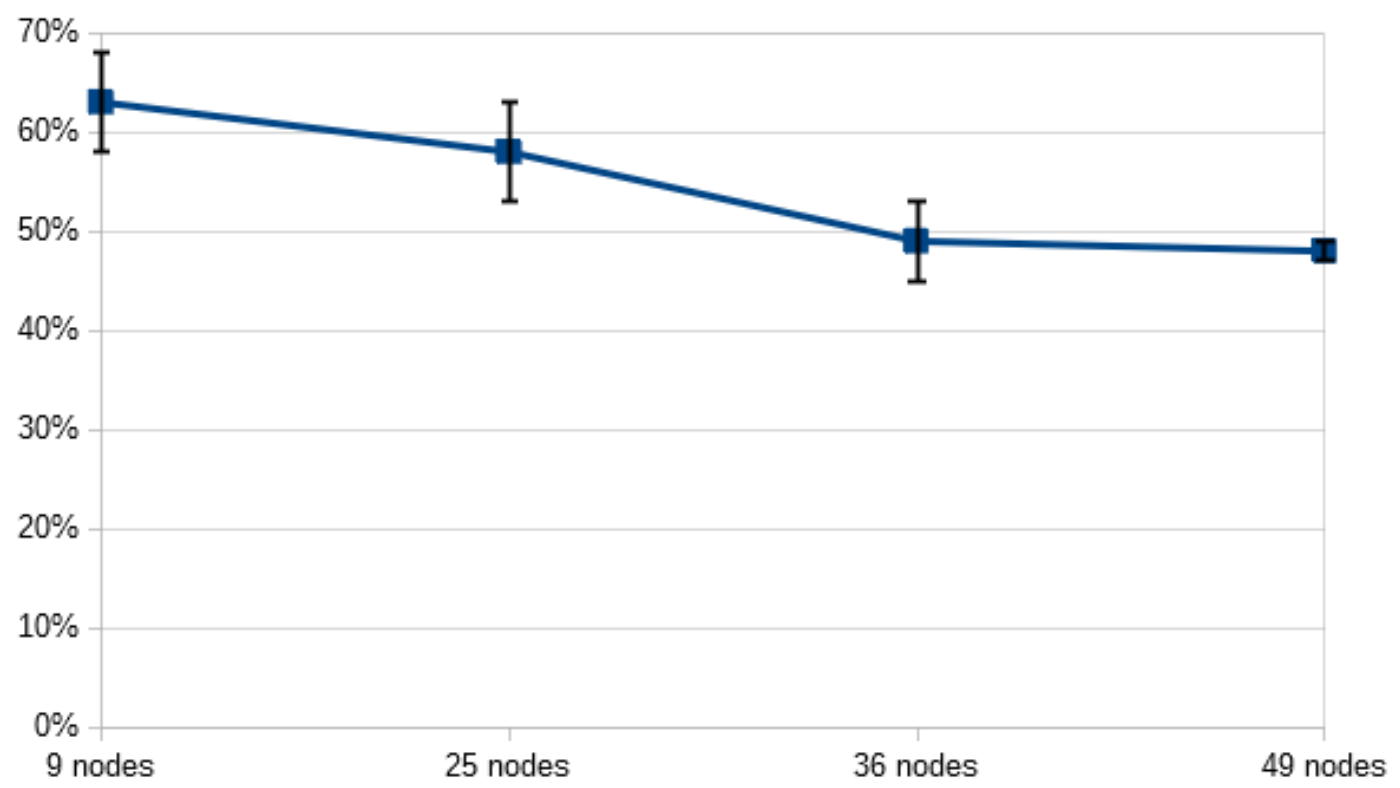

Figure 29: Delivery rate of AKA packets when establishing keys with the controller, for different network sizes. These results are from the 128-bits architecture.

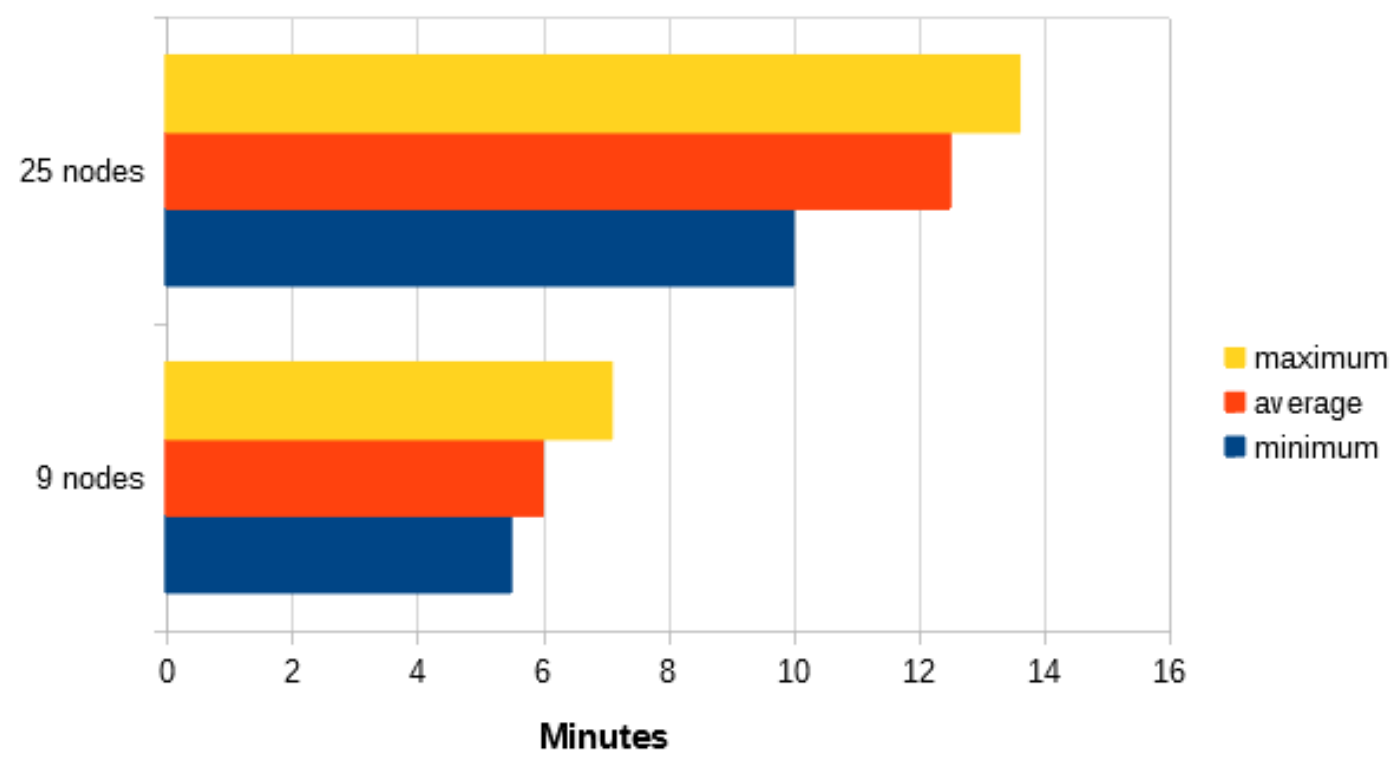

Figure 30: Time taken for the network to perform the AKA with the controller. These results are from the 128 bits architecture.

the memory of the sink node. We limit to 16 nodes the total amount of nodes in which the sink can store a key on its internal memory (while the 80 bits framework was possible to store 20 key entries, in our tests, 16 entries was the limit that a sink node 
Table 7: Parameters summary for the simulations with the 128-bits framework with a Sink node.

\begin{tabular}{ll}
\hline Parameter & Value \\
\hline Number of Nodes & $9,25,36$ \\
Topology & Grid with controller and sink at the center \\
Traffic Rate & IT-SDN default (5 seconds per packet) \\
Simulation Time & 20 Minutes \\
Simulation Tests & 5 times \\
\hline
\end{tabular}

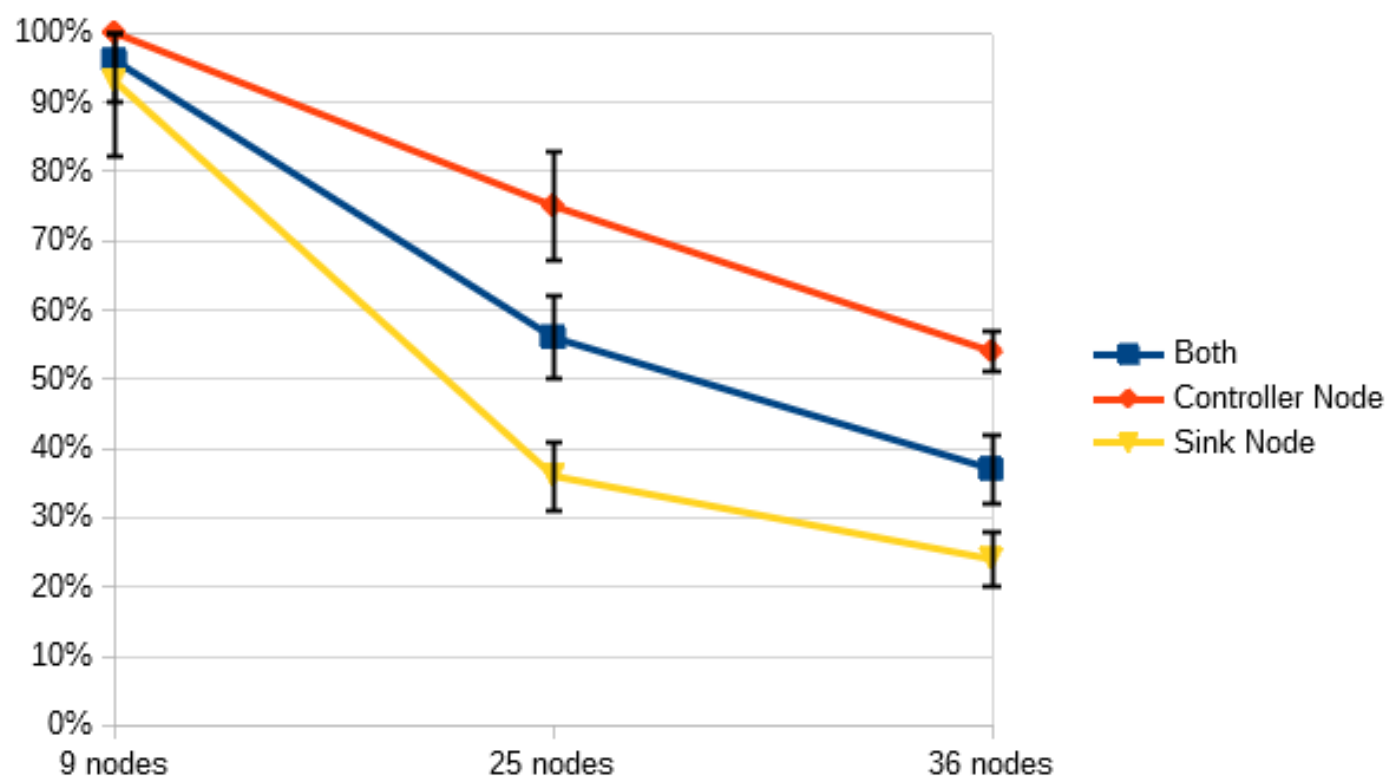

Figure 31: AKA completion rate for the network with a single sink and a single controller, for different numbers of nodes and an 128-bits security level. The curves represent the percentage nodes the controller; sink; and both completed the AKA process.

could store before an overflow occurred on its memory). This limitation, however, did not affect the results, as the 20 minutes of simulation time was not enough for the sink to complete 16 AKAs. Lastly before we proceed, the standard deviation of most results was of no more than $6 \%$, except of one particular result; the sink AKA completion rate with the network for the 25 nodes scenario, which showed a $11 \%$ standard deviation.

Figure 31 shows the completion rate of the AKA protocol. Due to the iSMQV protocol taking more than one minute to complete its procedures on the sink. Both scenarios of 25 and 36 nodes showed a decrease in the completion rate of the AKA in 


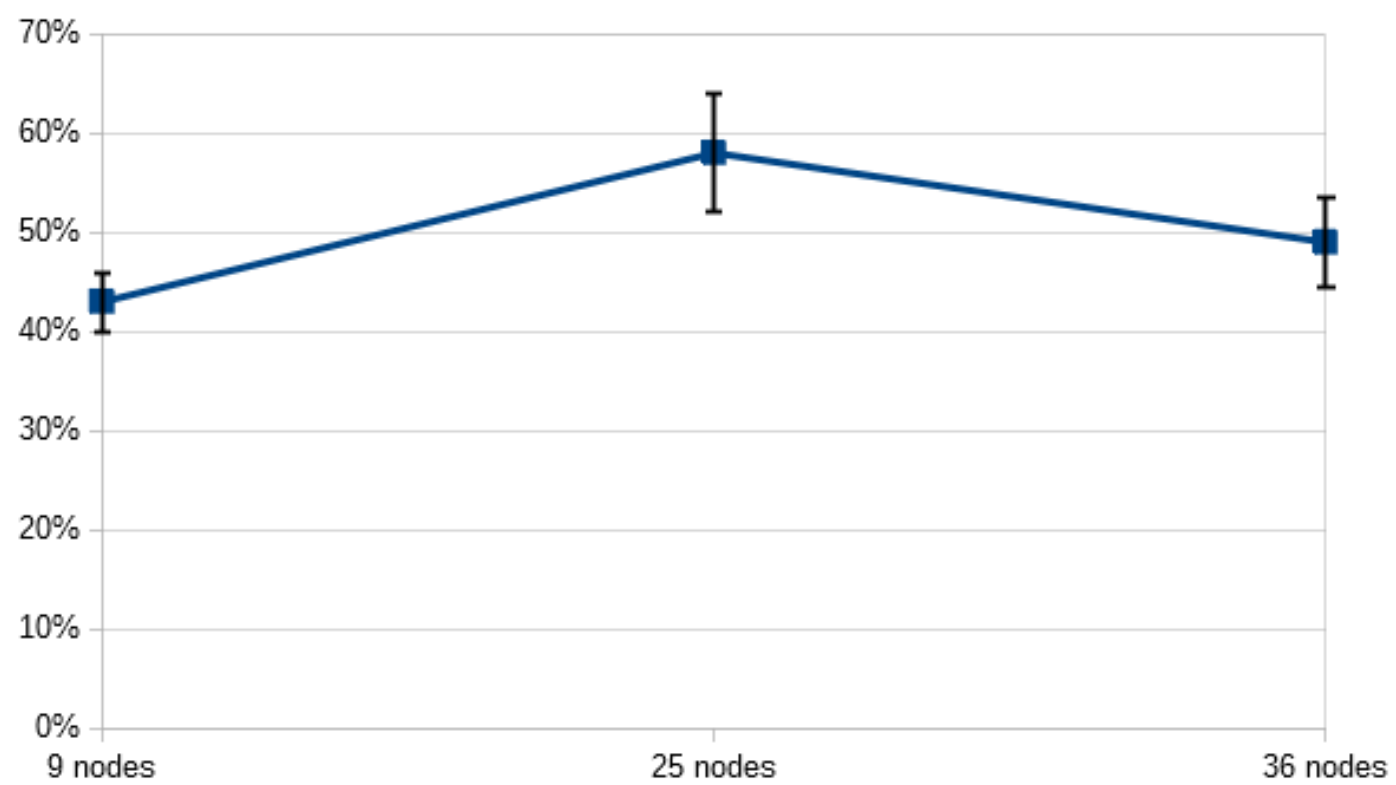

Figure 32: Delivery rate of AKA packets when establishing keys with the controller and sink, for different network sizes. These results are from the 128-bits architecture. Only the scenarios which completed the AKA during the simulation time are represented.

comparison to the scenario of 80 bits. While the scenario of 9 nodes managed to keep its completion rate during the stipulated simulation time.

Figure 32 shows the delivery rate of AKA procedure packets in the network. The small sized network of 9 nodes showed the worst results in terms of packet loss. This is likely due to the execution time of the iSMQV protocol. Since the network is small and less prone to packet loss due to packet collision, the AKA packets arrived early at the sink. However, due to the execution time of the iSMQV protocol, the sink could not answer these packets. In addition to this, the 9 nodes scenario managed to complete the AKA bootstrap during the simulation time, which was not the case for the 25 and 36 nodes scenario. So, it is not that the other scenarios showed a better delivery rate of such packets, but that they were not given the due time to complete these procedures. 


\section{FINAL CONSIDERATIONS}

In this work, we developed a comprehensive research on security implementation for SDWSN, investigating the state of art and taking a look at modern security solutions. After identifying limitations of WS3N as a security solution in the SDWSN scenario, and looking at the IT-SDN framework as a promising (albeit insecure) solution for building SDWSNs, we modeled the security services necessary for the latter's operation. Using as basis the iSMQV we present an architectural security solution for SDWSN. The ultimate goal of this solution is to provide authenticity, confidentiality and integrity to the packets of the framework, reducing attack surfaces and preventing relevant security threats.

Aiming to facilitate the deployment of these security services in SDWSNs, in this article we describe a security framework designed specifically for such environments. The proposed solution builds upon lightweight cryptographic mechanisms to provide a variety of end-to-end security services for sensor nodes, sinks and controllers. It is particularly effective against falsification and eavesdropping attacks, as it enables authorized nodes to encrypt and authenticate packets whenever required.

By simulating the proposed solution this work showed the impact of lightweight cryptographic and SDWSN when put together. The bootstrap of the required keys for communications are long and heavy (which only escalated with a higher security level), which was expected prior to this work. By implementing a way to make these communications optional, we wanted to suppress the burden of these security require- 
ments. It is highly suggested that security guidelines be only enforced in a small part of the network.

Lastly, we would like to point out some future research directions that would improve this solution. First, it would be interesting to develop an east-westbound interface to manage the amount of keys a sink node can hold. This interface could inform the controller of the sinks capacity, for example. As a result, the controller could use this information in conjunction with the routing protocol, preventing the node from starting an AKA with an overloaded sink. Another direction to take is to design a routing protocol that takes into account which nodes have a valid key stored in the controller database. This routing protocol could forward application data only (or preferably) through secure paths, reducing even more the attacks surface of the network.

\subsection{Articles Published}

During this research two publications were planned. One was published in the year of 2019 in the national conference of communications, network and information security (ENCOM). While another is completed and was submitted for publication. The Table 8 presents a summary of the publication plan, including their status.

Table 8: Publications during the research

\begin{tabular}{lll}
\hline Publication Title & Target & Status \\
\hline $\begin{array}{lll}\text { A Framework for Building Secure Software-Defined } \\
\text { Wireless Sensor Networks }\end{array}$ & Conference & Published \\
\hline $\begin{array}{l}\text { Enabling security in software-defined wireless } \\
\text { sensor networks for internet of things }\end{array}$ & Conference & Submitted \\
\hline
\end{tabular}




\section{REFERENCES}

AKYILDIZ, I. F.; WANG, P.; LIN, S.-C. Softwater: Software-defined networking for next-generation underwater communication systems. Ad Hoc Networks, Elsevier, v. 46, p. 1-11, 2016.

AL AMEEN, M.; LIU, J.; KWAK, K. Security and privacy issues in wireless sensor networks for healthcare applications. Journal of medical systems, Springer, v. 36, n. 1, p. 93-101, 2012.

ALI-AHMAD, H.; CICCONETTI, C.; OLIVA, A. De la; MANCUSO, V.; SAMA, M. R.; SEITE, P.; SHANMUGALINGAM, S. An sdn-based network architecture for extremely dense wireless networks. In: IEEE. Future Networks and Services (SDN4FNS), 2013 IEEE SDN for. [S.1.], 2013. p. 1-7.

ALQALLAF, M.; WANG, B. Software defined collaborative secure ad hoc wireless networks. In: IEEE. Collaboration Technologies and Systems (CTS), 2015 International Conference on. [S.1.], 2015. p. 196-203.

ALVES, R. C.; OLIVEIRA, D. A.; NÚÑEZ, G. A.; MARGI, C. B. It-sdn: Improved architecture for sdwsn. XXXV Simpósio Brasileiro de Redes de Computadores, 2017.

ALVES, R. C.; OLIVEIRA, D. A.; PEREIRA, G. C.; ALBERTINI, B. C.; MARGI, C. B. Ws3n: Wireless secure sdn-based communication for sensor networks. Security and Communication Networks, Hindawi, v. 2018, 2018.

ARANHA, D. F.; GOUVEA, C. P. L. RELIC is an Efficient LIbrary for Cryptography. https://github.com/relic-toolkit/relic.

ARANITI, G.; COSMAS, J.; IERA, A.; MOLINARO, A.; MORABITO, R.; ORSINO, A. Openflow over wireless networks: Performance analysis. In: IEEE. Broadband Multimedia Systems and Broadcasting (BMSB), 2014 IEEE International Symposium on. [S.1.], 2014. p. 1-5.

ATZORI, L.; IERA, A.; MORABITO, G. From" smart objects" to" social objects": The next evolutionary step of the internet of things. IEEE Communications Magazine, IEEE, v. 52, n. 1, p. 97-105, 2014.

AZZOUNI, A.; BOUTABA, R.; TRANG, N. T. M.; PUJOLLE, G. softdp: Secure and efficient topology discovery protocol for sdn. arXiv preprint arXiv:1705.04527, 2017.

BOSSHART, P.; DALY, D.; GIBB, G.; IZZARD, M.; MCKEOWN, N.; REXFORD, J.; SCHLESINGER, C.; TALAYCO, D.; VAHDAT, A.; VARGHESE, G. et al. P4: Programming protocol-independent packet processors. ACM SIGCOMM Computer Communication Review, ACM, v. 44, n. 3, p. 87-95, 2014. 
BROWN, D. R.; GALLANT, R.; VANSTONE, S. A. Provably secure implicit certificate schemes. In: SPRINGER. International Conference on Financial Cryptography. [S.1.], 2002. p. 156-165.

CAMPAGNA, M. Sec 4: Elliptic curve qu-vanstone implicit certificate scheme (ecqv). institution content-type $=$ institution $>$ Certicom Res $<$ institution $>$., Mississauga, ON, Canada, Tech. Rep, 2008.

CARDENAS, E. D. Mac spoofing-an introduction. GIAC Security Essentials Certification (GSEC), 2003.

CERTICOM. SEC 4 v1.0: Elliptic Curve Qu-Vanstone Implicit Certificate Scheme (ECQV). Canada, 2013. Disponível em: <http://www.secg.org/sec4-1.0.pdf > . Acesso em: $14 / 10 / 2020$.

CHEN, L.; BIAN, K. Neighbor discovery in mobile sensing applications: A comprehensive survey. Ad Hoc Networks, Elsevier, v. 48, p. 38-52, 2016.

CHIN, T.; MOUNTROUIDOU, X.; LI, X.; XIONG, K. An sdn-supported collaborative approach for ddos flooding detection and containment. In: IEEE. Military Communications Conference, MILCOM 2015-2015 IEEE. [S.1.], 2015. p. 659-664.

CUI, H.; KARAME, G. O.; KLAEDTKE, F.; BIFULCO, R. On the fingerprinting of software-defined networks. IEEE Transactions on Information Forensics and Security, IEEE, v. 11, n. 10, p. 2160-2173, 2016.

CUI, Y.; YAN, L.; LI, S.; XING, H.; PAN, W.; ZHU, J.; ZHENG, X. Sd-anti-ddos: Fast and efficient ddos defense in software-defined networks. Journal of Network and Computer Applications, Elsevier, v. 68, p. 65-79, 2016.

DE OLIVEIRA, B. T.; GABRIEL, L. B.; MARGI, C. B. Tinysdn: Enabling multiple controllers for software-defined wireless sensor networks. IEEE Latin America Transactions, IEEE, v. 13, n. 11, p. 3690-3696, 2015.

DHAWAN, M.; PODDAR, R.; MAHAJAN, K.; MANN, V. Sphinx: Detecting security attacks in software-defined networks. In: NDSS. [S.1.: s.n.], 2015.

DUNKELS, A.; GRONVALL, B.; VOIGT, T. Contiki-a lightweight and flexible operating system for tiny networked sensors. In: IEEE. 29th annual IEEE international conference on local computer networks. [S.1.], 2004. p. 455-462.

FLAUZAC, O.; GONZALEZ, C.; HACHANI, A.; NOLOT, F. Sdn based architecture for iot and improvement of the security. In: IEEE. Advanced Information Networking and Applications Workshops (WAINA), 2015 IEEE 29th International Conference on. [S.1.], 2015. p. 688-693.

FLOODLIGHT, P. FloodLight OpenFlow Controller. 2017. Disponível em: $<$ http://www.projectfloodlight.org/floodlight/>. Acesso em: 14/10/2020.

FRANKEL, S.; KRISHNAN, S. Ip security (ipsec) and internet key exchange (ike) document roadmap. [S.1.], 2011. 
GALLUCCIO, L.; MILARDO, S.; MORABITO, G.; PALAZZO, S. Sdn-wise: Design, prototyping and experimentation of a stateful sdn solution for wireless sensor networks. In: IEEE. Computer Communications (INFOCOM), 2015 IEEE Conference on. [S.1.], 2015. p. 513-521.

GREENE, K. Tr10: Software-defined networking. Technology Review (MIT), 2009.

GUPTA, V.; KAUR, K.; KAUR, S. Developing small size low-cost software-defined networking switch using raspberry pi. In: Next-Generation Networks. [S.1.]: Springer, 2018. p. 147-152.

HANKERSON, D.; MENEZES, A.; VANSTONE, S. Guide to Elliptic Curve Cryptography. [S.1.]: Springer-Verlag New York, Inc., 2003. ISBN 038795273X.

INSTRUMENT, T. MSP430F543xA, MSP430F541xA Mixed-Signal Microcontrollers. 2010. Disponível em: <https://www.ti.com/product/MSP430F5437A>. Acesso em: $14 / 10 / 2020$.

INSTRUMENT, T. MSP430 ${ }^{T M}$ ultra-low-power sensing measurement MCUs. 2010. Disponível em: <https://www.ti.com/microcontrollers/msp430-ultra-low-powermcus/overview.html>. Acesso em: 14/10/2020.

INSTRUMENT, T. Second generation 2.4 GHz ZigBee/IEEE 802.15.4 wireless transceiver. 2010. Disponível em: <https://www.ti.com/product/CC2520>. Acesso em: 14/10/2020.

JARARWEH, Y.; AL-AYYOUB, M.; BENKHELIFA, E.; VOUK, M.; RINDOS, A. et al. Sdiot: a software defined based internet of things framework. Journal of Ambient Intelligence and Humanized Computing, Springer, v. 6, n. 4, p. 453-461, 2015.

KAVITHA, T.; SRIDHARAN, D. Security vulnerabilities in wireless sensor networks: A survey. Journal of information Assurance and Security, v. 5, n. 1, p. 31-44, 2010.

KOBLITZ, N. Elliptic curve cryptosystems. Mathematics of computation, v. 48, n. 177, p. 203-209, 1987.

LIM, S.; YANG, S.; KIM, Y.; YANG, S.; KIM, H. Controller scheduling for continued sdn operation under ddos attacks. Electronics Letters, IET, v. 51, n. 16, p. 1259-1261, 2015.

LIN, P.; BI, J.; WOLFF, S.; WANG, Y.; XU, A.; CHEN, Z.; HU, H.; LIN, Y. A west-east bridge based sdn inter-domain testbed. IEEE Communications Magazine, IEEE, v. 53, n. 2, p. 190-197, 2015.

LUO, T.; TAN, H.-P.; QUEK, T. Q. Sensor openflow: Enabling software-defined wireless sensor networks. IEEE Communications letters, v. 16, n. 11, p. 1896-1899, 2012.

MAHMUD, A.; RAHMANI, R. Exploitation of openflow in wireless sensor networks. In: IEEE. Computer Science and Network Technology (ICCSNT), 2011 International Conference on. [S.1.], 2011. v. 1, p. 594-600. 
MARGI, C.; OLIVEIRA, B.; SOUSA, G.; SIMPLICIO, M.; BARRETO, P.; CARVALHO, T.; NÄSLUND, M.; GOLD, R. Impact of operating systems on wireless sensor networks (security) applications and testbeds. In: Proc. of the 19th International Conference on Computer Communication Networks (ICCCN'10) IEEE International Workshop on Wireless Mesh and Ad Hoc Networks (WiMAN'10). Washington, DC, USA: IEEE, 2010. p. 1-6. ISSN 1095-2055.

MARGI, C. B.; ALVES, R. C.; SEGURA, G. A. N.; OLIVEIRA, D. A. Softwaredefined wireless sensor networks approach: Southbound protocol and its performance evaluation. Open Journal of Internet Of Things (OJIOT), RonPub, v. 4, n. 1, p. 99-108, 2018.

MCKEOWN, N.; ANDERSON, T.; BALAKRISHNAN, H.; PARULKAR, G.; PETERSON, L.; REXFORD, J.; SHENKER, S.; TURNER, J. Openflow: enabling innovation in campus networks. ACM SIGCOMM Computer Communication Review, ACM, v. 38, n. 2, p. 69-74, 2008.

MEDVED, J.; VARGA, R.; TKACIK, A.; GRAY, K. Opendaylight: Towards a model-driven sdn controller architecture. In: IEEE. World of Wireless, Mobile and Multimedia Networks (WoWMoM), 2014 IEEE 15th International Symposium on a. [S.1.], 2014. p. 1-6.

MILLER, V. S. Use of elliptic curves in cryptography. In: SPRINGER. Conference on the theory and application of cryptographic techniques. [S.1.], 1985. p. 417-426.

MO, Y.; GARONE, E.; CASAVOLA, A.; SINOPOLI, B. False data injection attacks against state estimation in wireless sensor networks. In: IEEE. Decision and Control (CDC), 2010 49th IEEE Conference on. [S.1.], 2010. p. 5967-5972.

MOHAMMADI, R.; JAVIDAN, R.; CONTI, M. Slicots: An sdn-based lightweight countermeasure for tcp syn flooding attacks. IEEE Transactions on Network and Service Management, IEEE, 2017.

MONTENEGRO, G.; KUSHALNAGAR, N.; HUI, J.; CULLER, D. Transmission of IPv6 packets over IEEE 802.15.4 networks. [S.1.], 2007.

National Institute of Standards and Technology. Recommendation for Pair-Wise Key Establishment Schemes Using Discrete Logarithm Cryptography. 2013.

NGAI, E. C.; LIU, J.; LYU, M. R. On the intruder detection for sinkhole attack in wireless sensor networks. In: CITESEER. ICC. [S.1.], 2006. v. 6, p. 3383-3389.

NGUYEN, T.-H.; YOO, M. Analysis of link discovery service attacks in sdn controller. In: IEEE. Information Networking (ICOIN), 2017 International Conference on. [S.1.], 2017. p. 259-261.

OSTERLIND, F.; DUNKELS, A.; ERIKSSON, J.; FINNE, N.; VOIGT, T. Cross-level sensor network simulation with cooja. In: IEEE. Local computer networks, proceedings 2006 31st IEEE conference on. [S.1.], 2006. p. 641-648. 
PAKZAD, F.; PORTMANN, M.; TAN, W. L.; INDULSKA, J. Efficient topology discovery in software defined networks. In: IEEE. Signal Processing and Communication Systems (ICSPCS), 2014 8th International Conference on. [S.1.], 2014. p. 1-8.

PAULISTA, U. E. Kytos. 2017. Disponível em: <https://docs.kytos.io/>. Acesso em: $14 / 10 / 2020$.

PELECHRINIS, K.; ILIOFOTOU, M.; KRISHNAMURTHY, S. V. Denial of service attacks in wireless networks: The case of jammers. IEEE Communications surveys $\mathcal{E}$ tutorials, Institute of Electrical and Electronics Engineers, Inc., 3 Park Avenue, 17 th Fl New York NY 10016-5997 USA, v. 13, n. 2, p. 245-257, 2011.

PICKETT, G. Abusing software defined networks. BlackHat EU’14, 2014.

PORRAS, P.; SHIN, S.; YEGNESWARAN, V.; FONG, M.; TYSON, M.; GU, G. A security enforcement kernel for openflow networks. In: ACM. Proceedings of the first workshop on Hot topics in software defined networks. [S.1.], 2012. p. 121-126.

PRITCHARD, S. W.; HANCKE, G. P.; ABU-MAHFOUZ, A. M. Security in software-defined wireless sensor networks: Threats, challenges and potential solutions. In: IEEE. Industrial Informatics (INDIN), 2017 IEEE 15th International Conference on. [S.1.], 2017. p. 168-173.

RESEARCH, C. SEC 2: Recommended Elliptic Curve Domain Parameters. [S.1.], 2000 .

ROUSSEL, K.; SONG, Y.-Q.; ZENDRA, O. Using cooja for wsn simulations: Some new uses and limits. In: Proceedings of the 2016 International Conference on Embedded Wireless Systems and Networks. USA: Junction Publishing, 2016. (EWSN '16), p. 319-324. ISBN 9780994988607.

SAHOO, K. S.; SAHOO, B.; PANDA, A. A secured sdn framework for iot. In: IEEE. Man and Machine Interfacing (MAMI), 2015 International Conference on. [S.1.], 2015. p. 1-4.

SAKAI, R.; OHGISHI, K.; KASAHARA, M. Cryptosystems based on pairing. Cryptosystems Based on Pairing, p. 26-28, 2000. Cited By :33. Disponível em: <www.scopus.com>. Acesso em: 14/10/2020.

SARR, A. P.; ELBAZ-VINCENT, P.; BAJARD, J.-C. A new security model for authenticated key agreement. In: SPRINGER. International Conference on Security and Cryptography for Networks. [S.1.], 2010. p. 219-234.

SCHMID, S.; SUOMELA, J. Exploiting locality in distributed sdn control. In: ACM. Proceedings of the second ACM SIGCOMM workshop on Hot topics in software defined networking. [S.1.], 2013. p. 121-126.

SCOTT-HAYWARD, S.; KANE, C.; SEZER, S. Operationcheckpoint: Sdn application control. In: IEEE. Network Protocols (ICNP), 2014 IEEE 22nd International Conference on. [S.1.], 2014. p. 618-623. 
SHAGHAGHI, A.; KANHERE, S. S.; KAAFAR, M. A.; JHA, S. Gwardar: Towards protecting a software-defined network from malicious network operating systems. arXiv preprint arXiv:1809.06988, 2018.

SHARMA, K.; GHOSE, M. Wireless sensor networks: An overview on its security threats. IJCA, Special Issue on Mobile Ad-hoc Networks MANETs, p. 42-45, 2010.

SHERWOOD, R.; GIBB, G.; YAP, K.-K.; APPENZELLER, G.; CASADO, M.; MCKEOWN, N.; PARULKAR, G. Flowvisor: A network virtualization layer. OpenFlow Switch Consortium, Tech. Rep, v. 1, p. 132, 2009.

SIMPLICIO, M.; BARRETO, P.; MARGI, C.; CARVALHO, T. A survey on key management mechanisms for distributed wireless sensor networks. Computer Networks, Elsevier, Amsterdam, The Netherlands, v. 54, n. 15, p. 2591-2612, 2010. ISSN 1389-1286.

SIMPLICIO, M.; OLIVEIRA, B.; MARGI, C.; BARRETO, P.; CARVALHO, T.; NÄSLUND, M. Survey and comparison of message authentication solutions on wireless sensor networks. Ad Hoc Networks, Elsevier, Amsterdam, The Netherlands, v. 11, n. 3, p. 1221-1236, maio 2013. ISSN 1570-8705.

SIMPLICIO, M.; OLIVEIRA, B.; MARGI, C.; BARRETO, P.; CARVALHO, T.; NÄSLUND, M. Survey and comparison of message authentication solutions on wireless sensor networks. Ad Hoc Networks, Elsevier, Amsterdam, The Netherlands, v. 11, n. 3, p. 1221-1236, maio 2013. ISSN 1570-8705.

SIMPLICIO JR., M. A.; BARBUDA, P. d. F. F. S.; BARRETO, P. S. L. M.; CARVALHO, T. C. M. B.; MARGI, C. B. The marvin message authentication code and the lettersoup authenticated encryption scheme. Security and Communication Networks, v. 2, n. 2, p. 165-180, 2009. Disponível em: $<$ https://onlinelibrary.wiley.com/doi/abs/10.1002/sec.66>. Acesso em: 14/10/2020.

SIMPLICIO JR, M. A.; SILVA, M. V.; ALVES, R. C.; SHIBATA, T. K. Lightweight and escrow-less authenticated key agreement for the Internet of Things. Computer Communications, Elsevier, v. 98, p. 43-51, 2017. ISSN 0140-3664.

SON, S.; SHIN, S.; YEGNESWARAN, V.; PORRAS, P.; GU, G. Model checking invariant security properties in openflow. In: IEEE. Communications (ICC), 2013 IEEE International Conference on. [S.1.], 2013. p. 1974-1979.

TADINADA, V. R. Software defined networking: Redefining the future of internet in iot and cloud era. In: IEEE. Future Internet of Things and Cloud (FiCloud), 2014 International Conference on. [S.1.], 2014. p. 296-301.

V1.2, A. Dobraunig, C. and Eichlseder, M. and Mendel, F. and Schläffer, M. 2016. Submission to the CAESAR competition: http://competitions.cr.yp.to/ round3/asconv12.pdf. See also: http://ascon.iaik.tugraz . at.

(V3), A. A. L. A. C. H. Wu. 2016. Submission to the CAESAR competition: https://competitions.cr.yp.to/round3/acornv3.pdf. 
WANG, X.; GAO, N.; ZHANG, L.; LIU, Z.; WANG, L. Novel mitm attacks on security protocols in sdn: A feasibility study. In: SPRINGER. International Conference on Information and Communications Security. [S.1.], 2016. p. 455-465.

WEN, X.; CHEN, Y.; HU, C.; SHI, C.; WANG, Y. Towards a secure controller platform for openflow applications. In: ACM. Proceedings of the second ACM SIGCOMM workshop on Hot topics in software defined networking. [S.1.], 2013. p. $171-172$.

WINTER, T.; THUBERT, P.; BRANDT, A.; HUI, J.; KELSEY, R.; LEVIS, P.; PISTER, K.; STRUIK, R.; VASSEUR, J. P.; ALEXANDER, R. RPL: IPv6 routing protocol for low-power and lossy networks. [S.1.], 2012. 